\title{
Controlling the Spectroscopic Properties of Quantum Dots via Energy Transfer and Charge Transfer Interactions: Concepts and Applications
}

\author{
Xin Ji, ${ }^{+, a}$ Wentao Wang, ${ }^{+}$Hedi Mattoussi
}

Department of Chemistry and Biochemistry, Florida State University, 95 Chieftan Way, Tallahassee, Florida 32306, United States

Keywords: Quantum Dots, Confinement Effects, Fluorescence, Fluorescence Resonance Energy Transfer (FRET), Charge Transfer (CT), Biosensing.

\begin{abstract}
Summary
Luminescent quantum dots (QDs) exhibit size- and composition-tunable photophysical properties that are not shared by their bulk parent materials or at the molecular scale. They have attracted considerable interest further motivated by several potential applications. A particular interest has centered on exploiting the ability of QDs to engage in both fluorescence resonance energy transfer (FRET) and charge transfer (CT) interactions with proximal fluorophores and redox active molecules/complexes, respectively. In this review, we highlight how the QD's optical and spectroscopic properties can be controlled via FRET and/or CT interactions. We first show that QDs provide a unique platform for controlling both modes of interactions. We then provide representative examples in biology which include developing sensing assemblies that report on properties such as $\mathrm{pH}$ changes, enzymatic activity and ligandreceptor binding. Implications in electronic devices focus on light emitting devices and photovoltaic cells, where we discuss device architecture, control over carrier injection, carrier mobility, and exciton recombination.
\end{abstract}

\footnotetext{
Authors contributed equally to the report

a Present address: Ocean Nanotech, LLC, 7964 Arjons Drive, San Diego, CA 92126

Email: mattoussi@chem.fsu.edu
} 


\section{Introduction}

Colloidal nanoparticles including semiconductor nanocrystals, metal and metal oxide nanoparticles have generated tremendous interests in the past two decades, due to some of their unique fundamental properties and the potential they offer for a variety of applications.[1-20] These materials possess several photophysical properties that can be tuned by size and/or composition.[1, 2, 8, 9] Luminescent semiconductor nanocrystals (quantum dots, QDs), for example, exhibit remarkable photo stability, broad absorption profiles with large oneand two-photon cross-section, narrow tunable emission spectra and high quantum yield.[1, 2, 21-28] This makes them potentially useful in optical and electronic devices, such as light emitting diodes and photovoltaic devices.[29-35] These applications have been supplemented by a large potential for use as in vivo and in vitro probes in a variety of biomedical applications.[3, 5-7, 14, 16-19, 36]

One very important feature of colloidal nanocrystals is their large surface-to-volume ratio, resulting in a sizable fraction of their atoms arrayed on their surfaces. As such their optical and electronic properties are highly sensitive to the nature of the surface ligands, namely, coordination interactions, lateral extension and electronic properties. The photophysical properties of the nanocrystals can also be strongly influenced by interactions with proximal redox complexes and dyes via charge transfer and/or energy transfer. This offers additional means to modulate the QD emission and carrier dynamics.[20, 37-41]

Fluorescence resonance energy transfer (FRET) and charge transfer (CT) interactions are two photophysical processes that are known to alter the absorption and emission properties of conventional fluorophores and they can equally affect the properties of QDs (Figure 1). Such interactions provide a means to externally control the optical and spectroscopic properties of QDs. They have been exploited by various research groups to develop sensing platforms based on QDs that can allow the detection of target analytes, probing $\mathrm{pH}$ changes and accounting for ligand-receptor binding.[5, 16, 19, 20, 36, 42-44] Similarly, the above optical and spectroscopic properties exhibited by QDs have motivated their effective use for deep tissue imaging and as drug delivery vehicles.[6, 16-19, 36, 45-47] 
There are a few parameters that can affect and control FRET and CT interactions: (1) size and shape of the nanocrystal, (2) spectral overlap between donor emission and acceptor absorption profiles, (3) the separation distance, and (4) the number of acceptors and their orientations around a single QD; the latter is particularly important for QDs because of their nanoscale dimensions. Such interactions can also be probed using relatively simple experimental techniques, such as steady-state and time-resolved fluorescence spectroscopy.[48-52] Transient absorption (TA) and transient florescence spectroscopy techniques can also be used to assess electron and/or hole transfer and dynamics in these complexes.[40, 53-55]

In this report, we have assembled a short overview of ideas and rationales developed over the past decade for modulating the photophysical and electronic properties of quantum dots. We start by describing the fundamental concepts governing energy and charge transfer interactions. We then describe a few examples showing exquisite control over the QD fluorescence quenching using either FRET and/or CT interactions, along with implementations in biological sensing. A few examples showing the importance of charge transfer interactions between QDs and other nanomaterials with implications in light emitting devices and solar cells will also be discussed.

\section{Resonance Energy Transfer and Charge Transfer Interactions: Background}

\subsection{FRET Interactions}

FRET is a process that involves the non-radiative transfer of excitation energy from a photoexcited dye (donor, D) to a nearby ground state dye (acceptor, A). Such interactions manifest in a loss of the donor emission coupled with enhanced acceptor fluorescence; if the acceptor is non-fluorescent, only quenching of the donor emission is observed following interactions. FRET interactions also manifests in shortening of the donor fluorescence lifetime. Within the Förster formalism FRET results from coupling between the donor and acceptor dipoles (developed by Förster), and as such it strongly depends on the center-to-center separation distance. $[39,56]$ To produce efficient FRET interactions, a few key criteria must be satisfied, including: (a) close proximity between a donor and an acceptor (i.e., short separation 
distance, $r$, due to the nature of the dipole-dipole coupling); (b) finite (nonzero) overlap between the donor emission and acceptor absorption spectra. In addition to those two requirements, FRET also depends on the orientation between donor and acceptor dipoles, with maximum coupling measured for parallel diploes, but no interactions measured for orthogonal dipoles.

The rate of energy transfer between a donor and an acceptor separated by a center-tocenter distance $r$, is defined as:[39, 56]

$$
k_{F R E T}=\frac{1}{\tau_{D}}\left(\frac{R_{0}}{r}\right)^{6}
$$

where $\tau_{D}$ designates the average exciton lifetime measured for the donor in the absence of an acceptor, and the Förster radius, $R_{0}$, corresponding to a separation distance at which the FRET rate is equal to the natural decay rate, $1 / \tau_{D} ; R_{0}$ is expressed as:

$$
R_{0}=\left(\frac{9000 \times \ln (10) \times k_{p}^{2} Q_{D} I}{128 \pi^{5} n_{D}{ }^{4} N_{A}}\right)^{1 / 6}
$$

In Eq. 2, $n_{D}$ designates the refractive index of the medium, $Q_{D}$ is the donor PL quantum yield, $N_{A}$ is the Avogadro's number, $k_{p}^{2}$ is the orientation factor $\left(k_{p}^{2}=2 / 3\right.$ for randomly oriented donor and acceptor dipoles), and I is the spectral overlap integral. The latter is defined in terms of the spectral overlap function, $J(\lambda)$, as:

$$
I=\int J(\lambda) d \lambda
$$

with $\quad J(\lambda)=\frac{F_{D}(\lambda) \varepsilon_{A}(\lambda) \lambda^{4}}{\int F_{D}(\lambda) d \lambda}=P L_{D-\text { corr }}(\lambda) \varepsilon_{A}(\lambda) \lambda^{4}$

where $P L_{D \text {-corr }}$ is the normalized donor emission spectrum and $\epsilon_{A}(\lambda)$ is the acceptor extinction coefficient spectrum. The overlap integral provides a quantitative measure of the donor and acceptor spectral overlap over all wavelengths.

The energy transfer efficiency is extracted from the above rate (Eq. 1) using: 


$$
E_{F R E T}=\frac{k_{D-A}}{k_{D-A}+\frac{1}{\tau_{D}}} \text {, which becomes } E_{F R E T}=\frac{R_{0}{ }^{6}}{R_{0}{ }^{6}+r^{6}}
$$

Within this definition, $R_{0}$ designates the separation distance corresponding to $50 \%$ FRET efficiency. FRET interactions are accounted for experimentally by measuring the energy transfer efficiency, $E$, often referred to as the PL quenching efficiency, using either steady-state or timeresolved fluorescence data via the expressions:[56]

$$
E=1-\frac{F_{D A}}{F_{D}}, \quad \text { for steady-state fluorescence }
$$

$$
\text { and } \quad E=1-\frac{\tau_{D A}}{\tau_{D}}, \quad \text { for time-resolved fluorescence }
$$

Here, $F_{D A}$ and $F_{D}$ respectively designate the ensemble $\mathrm{PL}$ intensities measured for the sample containing D-A pair and the control sample made of donors without any acceptors.

\subsection{Charge Transfer Interactions}

In contrast to FRET, charge transfer interactions do not always involve "fluorescent" molecules, as they occur in a variety of electronic processes including photovoltaic and light emitting devices. We focus on CT interactions between distinct molecules/complexes/species, not intramolecular (i.e., not occurring within distinct regions of the same molecule). These interactions involve the transfer of electrons and/or holes between a "donor" and an "acceptor" pairs. This process strongly depends on the separation distance (as does FRET, though more strongly), and requires the proper mismatch of the energy levels between the donor and the acceptor. In particular, electron transfer depends on a drop in the energy levels of the conduction bands (or LUMO levels) from the donor to the acceptor. Conversely, hole transfer (which is essentially reverse electron transfer) requires an increase in the valence band energies (or HOMO levels) from the donor to the acceptor.

The most effective theoretical treatment of CT interactions is the model of electron-

transfer between two states developed by R. Marcus in 1956.[57] This treatment has successfully described photoinduced electron transfer processes for a wide variety of systems 
and materials.[58-61] Furthermore, it was applied to the description of electron transfer interactions between a single donor state and a continuum of acceptor states (such as the case for the conduction and valence bands of semiconductor quantum dots).[62-64] Key factors that control the rate of charge transfer $\left(k_{C T}\right)$ include: the distance between the donor and acceptor complexes; the Gibbs energy of activation $(\Delta G)$; the system reorganization energy $(\lambda)$ and the electronic coupling strength $(H)$ between donor and acceptor sites. Focusing on interactions involving electrons, the transfer rate from a single donor state to a continuum of acceptor states can be expressed as:[64]

$$
k_{C T}=\frac{2 \pi}{\hbar} \frac{H^{2}}{\sqrt{4 \pi \lambda k_{B} T}} e^{\left\{-\frac{\left(\Delta G+\lambda^{2}\right)}{4 \lambda k_{B} T}\right\}}
$$

where $k_{B}$ and $\hbar$ designate the Boltzmann constant and the Planck constant, respectively, while $T$ is absolute temperature. The change in free energy $(\Delta G)$ of the system is associated with the mismatch between the energy levels of the donor and acceptor. The parameter $H$ describes the electronic coupling strength between the donor and acceptor states, and accounts for the dependence of $k_{C T}$ on the center-to-center separation distance. The electronic coupling strength is predicted to have an exponential dependence on the separation distance, $r:[37,57,65-67]$

$$
H^{2}=H_{0}^{2} e^{-\beta r}
$$

where $H_{0}$ is an electronic factor and $\beta$ is a constant that primarily depends on the nature of the bridge molecule. For a system where all the parameters are fixed except the electronic coupling $H^{2}$, the expression for $k_{C T}$ yields:[52]

$$
\ln \left(k_{C T}\right)=-\beta r+k_{0}
$$

Here, $k_{0}$ is a prefactor that depends on the relative alignment of energy levels of the redox complexes with respect to the energy levels of the donor $(\Delta G)$. The separation distance can be controlled physically by choosing the size of the bridge, e.g., using a linear poly(ethylene glycol) with different molecular weights, or a DNA double strand with varying numbers of base-pairs. This theory has been used to describe photoinduced electron transfer processes from QDs to an array of materials. $[63,64]$ 
One of the challenges facing researchers when probing CT processes is to identify how electron (or hole) shuttle between the donor and acceptor, i.e., do CT interactions require a bridge entity or they can occur only through space? Ratner and co-workers proposed a donorbridge-acceptor (D-B-A) framework,[68] where the bridge functions as a spacer between the pair, possibly comprising ligands (e.g., for QDs), linkers or inorganic shell (in core-shell nanostructures, as shown in Figure 1). The charge transfer rate constant $k_{C T}$ for a donoracceptor pair separated by a bridge can be experimentally extracted from time-resolved fluorescence measurements for fluorescent donors (such as QDs) using:[63, 69, 70]

$$
k_{C T}=\frac{1}{\tau_{D A}}-\frac{1}{\tau_{D}}
$$

where $\tau_{D A}$ and $\tau_{D}$ respectively designate the average PL lifetimes measured for dispersions of the donor-acceptor pair and for donor alone (control). As done above for FRET, an expression for the quenching efficiency can be derived for CT-induced PL loss:

$$
E_{C T}=\frac{k_{C T}}{k_{C T}+\frac{1}{\tau_{D}}}
$$

Here, we introduced a nonradiative relaxation channel for the excitation energy due to CT, with a rate proportional to the $k_{\mathrm{CT}}$ that is competing with the radiative decay rate $1 / \tau_{\mathrm{D}}$.

\section{QD Photo-physical Properties that are Beneficial to FRET and CT Interactions}

The strong dependence of FRET and CT processes on the D-A separation, $r$, makes them well suited for probing interactions that occur over separation distances of 10-100Å. FRET for example is ideally suited for assessing the size of biomolecules (proteins, DNA as well as synthetic peptides), changes in protein or DNA conformation promoted by interactions with target molecules or change in the surrounding conditions, and ligand-receptor binding. Similarly, CT has been widely used to probe proximity interactions between dyes and redox active complexes. Sensing based on FRET or CT employing conventional fluorophores has been widely used to provide real-time information on specific events, such as calcium signaling, nutrient flux and protein folding. One of the limitations encountered by dyes used in designing 
FRET-sensing constructs has been the difficulty to optically separate the emissions of donor and acceptor fluorophores. This is due to the rather broad emission and narrow absorption profiles combined with modest extinction coefficients of conventional dyes and fluorescent proteins. This makes exciting the donor only and separating the donor and acceptor signals somewhat tricky.[71] In addition, the narrow absorption profiles make it difficult to efficiently excite multiple dyes with a single laser line.

In recent years, interest in using QDs to develop a variety of sensors and devices based on FRET and CT interactions has steadily grown. $[35,49,63,72]$ This is motivated by some of the ability to tune the photophysical and chemical properties of colloidal QDs. Unlike bulk semiconductors, the energy levels of a QD can be adjusted/controlled, due to the sizedependent confinement of the electron-hole carriers within the physical dimensions of the nanocrystal.[73-75] Such confinement arises when the size of the nanocrystal decreases below the Bohr exciton radius of the material. The effects of carrier confinement disrupt the "continuum" in energy states, resulting in the appearance of discrete energy levels for the electron (conduction band) and hole (valence band). This yields a set of size- and compositiondependent features that are not shared by either their parent-bulk materials or at the molecular scale. Indeed, fluorescent QDs offer an ideal platform where the optical and electronic properties can be manipulated by size. For example, when a nanocrystal size is reduced (for a given composition), the optical band gap widens and the continuum in the energy distribution for the electron and hole (e-h pairs or excitons) is broken, yielding a set of size-tunable energy levels, along with blue shift in the absorption and emission spectra.[1, 13, 55] This allows control over the strength of the interactions in QD systems relying on either FRET or CT processes. For instance, several features exhibited by QDs can benefit FRET applied to either ensemble or single molecule measurements. Below we summarize these attributes for using QDs as donors to develop sensing configurations based on either FRET or CT interactions, where the level of coupling and modulation of the QD fluorescence can be controlled and tuned. Here, we mainly focus on a few key factors that permit control over the optical and electronic properties of QDs. 


\subsection{Controlling the Spectral Overlap by Varying the QD Emission}

By tuning the QD emission (e.g., by changing its core radius), one can optimize the spectral overlap function/integral shown in Eq. 3. Two examples demonstrating this capability are illustrated in Figure 2A. The first shows the change in the spectral overlap between the absorption of Cy5 dye and the emissions of two different-size CdSe-ZnS QDs, one emitting at $540 \mathrm{~nm}$ and the other at $592 \mathrm{~nm}$ (see areas highlighted in pink and purple); the overlap increases when the nanocrystal emission is closer to the Cy5 absorption maximum. In the second we show plots of the Cy3 absorption together with the PL from three different-size CdSe-ZnS QDs, along with the corresponding spectral overlap functions (see Figure 2A, right).[76] Tuning and optimizing the spectral overlap for a given donor-acceptor pair is made easier by the narrow and symmetric emission of the QDs. This in turn allows one to increase the experimental FRET efficiency for a given dye, by choosing the QD with the optimal overlap.[76]

\subsection{Tuning the Energy Mismatch by Changing the QD Size}

Changing the QD size alters the energy levels (in both the valence and conduction bands) and changes the energy band gap. This is beneficial for controlling and optimizing the rate of charge transfer (electron as well as hole) from a photo-excited QD to a proximal redox active complex.[51] This can also affect the rate of electron injection into a non-excited QD, which is known to affect the bleaching of the first exciton peak.[29] Figure 2B shows a schematic representation of the effects of varying the energy levels on the charge transfer interactions (involving electron or/and hole carriers) for two distinct-size QDs: green QDs with larger bandgap and red QDs with smaller bandgap. Two pathways are illustrated: (1) electron transfer and (2) hole transfer from a photoexcited QD to a redox complex; the latter can also be viewed as electron transfer from the complex to the empty valence band of a photoexcited QD. Here, the changes in the QD size alter the rate of electron-hole radiative recombination dynamics; faster rates are expected for QD-redox complexes prepared using smaller size QDs due to the larger energy bandgap.[51] 


\subsection{Tuning the Interactions by Controlling the Conjugate Valence}

This is perhaps the most important advantage offered by QDs in both FRET and CT interactions. Because of the large surface-to-volume ratio of the nanocrystals, a single QD can simultaneously interact with several proximal dyes or redox active complexes. This yields a configuration where the QD acts as a scaffold for immobilizing multiple molecules and as a donor simultaneously interacting with several acceptors via FRET, or as CT center interacting with several redox active molecules. This configuration produces a substantial increase in the "cross-sectional" interactions of the central QD with the surrounding dyes or redox complexes. When considering energy transfer this multichannel interaction of a QD with the surrounding dyes can produce a large increase in the FRET rate and the corresponding quenching efficiency, compared to a simple one-donor-one-acceptor pair. The overall energy transfer rate is the summation over all individual rates:

$$
k_{F R E T / n}=\sum_{i=1}^{n} k_{D-A, i}
$$

leading to an energy transfer efficiency that can be expressed as:

$$
E_{n}=\frac{k_{F R E T / n}}{k_{F R E T / n}+\tau_{D}^{-1}}=\frac{\sum_{i=1}^{n} k_{D-A, i}}{\sum_{i=1}^{n} k_{D-A, i}+\tau_{D}^{-1}}
$$

Here, each individual energy transfer rate $k_{D-A, i}$ within the QD-dye assembly depends on the spectral overlap integral (shown in Eq. 3), $n$ is the total number of acceptors interacting with the same donor, and $\tau_{D}$ is the PL lifetime of the donor alone. For a configuration where $n$ identical acceptors are arrayed at a given distance (i.e., $r$ is now fixed for all acceptors) around a central $Q D$, the efficiency can be further simplified to:

$$
E=\frac{n k_{D-A}}{n k_{D-A}+\tau_{D}^{-1}}=\frac{n R_{0}^{6}}{n R_{0}^{6}+r^{6}}
$$

The enhancement in FRET efficiency with increasing dye-to-QD ratio has been demonstrated in several studies using QD-dye and QD-fluorescent protein conjugates.[76-81] Figure 3A shows a schematic representation of a QD conjugated to several Cy3-labeled maltose binding proteins 
(MBP) via metal-polyhistidine self-assembly,[76] along with the evolution of the deconvoluted PL spectra collected from dispersions of those QD-conjugates with increasing protein-Cy3-toQD ratio $n$. The data shown in Figure 3A and in reference [76] clearly prove that there is a progressive quenching of the QD emission and a systematic enhancement of the dye acceptor emission with increasing conjugate valence. Similar data have been measured for QD-mCherry conjugates.[82]

Similar to FRET, the rate of charge transfer interactions is also increased by arraying several redox complexes around a central QD. Here too an expression for the CT rate can be written as:

$$
k_{C T / t o t}=\sum_{i=1}^{n} k_{C T, i}
$$

with $k_{\mathrm{CT}}$ given by Eq. 9. This produces an enhanced fluorescence quenching efficiency (e.g., for emitting donors such as QDs) that tracks the number of redox groups, with an expression in the form:

$$
E_{\text {quenching/ }}=\frac{\sum_{i=1}^{n} k_{C T, i}}{\sum_{i=1}^{n} k_{C T, i}+\tau_{D}^{-1}}
$$

which can become for centro-symmetric assembly:

$$
E_{\text {quenching/n }}=\frac{\sum_{1}^{n} k_{C T}}{\sum_{1}^{n} k_{C T}+\tau_{D}^{-1}}=\frac{\alpha n}{\alpha n+K}
$$

Where $\alpha$ is the parameter that depends on the relative alignment of the oxidation/reduction potential of the complex with respect to the energy levels of the QD (Eq. 6); $K$ strictly depends on the separation distance.[49]

Effects of conjugate heterogeneity. We should also note that conjugation of QDs (or any nanoparticles) with target molecules is a process that inherently yields a heterogeneous distribution in the number of attached molecules per QD-conjugate. Such distribution can affect the analysis of the quenching efficiency data (driven by FRET and CT interactions alike) 
and influences the comparison between experiment and theory. It has been proposed and experimentally verified that effects of the distribution in QD-acceptor conjugate valence on the measured efficiency data can be done using Poisson statistics, [83] where for a nominal valence $\mathrm{N}$ ( $\mathrm{N}$ being the experimental average number of acceptors per $\mathrm{QD}$ used), the exact number of acceptors ( $\mathrm{n}$ ) attached to a single QD varies following the Poisson expression: [83]

$$
p(n, N)=N^{n} \frac{e^{-N}}{n !}
$$

Thus, the quenching efficiency predicted for FRET (shown in Eq. 13) can be rewritten using the effects of heterogeneity in conjunction with the Poisson statistics as:

$$
E(N)=\sum_{n=1}^{N} p(n, N) E(n)
$$

A similar formula can be written for CT starting from Eq. 16. This analysis is crucial for studies requiring single QD fluorescence quenching measurements. They also help improving the data analysis when high levels of PL losses are measured for a sample using, for example, very short separation distances and/or large overlap integral.[83]

\subsection{Effects of Separation Distance}

Controlling the separation distance can, in principle, be altered by changing the QD radius. Shorter distance can be achieved using smaller size QDs, which should enhance the rate of FRET and CT interactions given the strong dependence of those processes on the center-tocenter separation distance. However, such effects are not isolated from those associated with tuning the spectral overlap (for FRET) or the energy mismatch (for CT), since any changes in QD size also strongly affects the energy levels. Separation distance can, however, be controlled via the size of the capping ligands, or linkers used to couple the target molecules to the QD surface. The range of ligand chemistries developed over the past several years provides an effective means for controlling the interactions and the ensued PL quenching. The schematics shown in Figure 3B depicts how the separation distance can be varied using 
different size bridges made of poly(ethylene glycol) segments with different molecular weights for a set of QD-PEG-dopamine conjugates.[52] The highest quenching efficiency was measured for the assembly with the shortest distance (see Figure 3B).[52]

The above features indicate that the use of QDs combined with FRET- and CT-induced quenching provides a great system for designing a variety of sensing assemblies. In addition, CT interactions play crucial roles in the operational efficiency of other devices where fluorescence is somewhat unimportant, namely photovoltaic devices. In the following sections, we will focus on several sensor designs based on CT or FRET processes. We will also discuss a few representative light emitting and photovoltaic devices, where controlled CT interactions play a key role in improving their performance.

\section{Sensing Based on FRET- and CT-induced Quenching of QD Emission}

We loosely define the notion of sensing as one's ability to either detect a target molecule or report on a biological process using a QD-conjugate assembly, where the signal transduction results from changes in the QD PL due to FRET and/or CT interactions. We classify the various sensors by a combination of the configuration employed and the nature of the targeted molecule or biological process.

\subsection{FRET- and CT-based pH Sensing}

Sensing of $\mathrm{pH}$ changes is critically important in many aspects of biological research. Subcellular organelles/compartments are often maintained at different $\mathrm{pH}$ from that measured in the surrounding environment. This necessitates that an active proton gradient is preserved to maintain the $\mathrm{pH}$ difference between the two compartments within a given range.[84] There are several intracellular processes, such as enzyme reaction and ATP synthesis/hydrolysis, which are controlled by changes in the local concentration of protons.[85] Thus, designing molecular assemblies that can allow accurate monitoring of local changes in $\mathrm{pH}$ is highly desired, and new $\mathrm{pH}$-sensing constructs continue to be sought. With the ability to control the QD PL signal by FRET, CT or both, researchers have pursued the ideas of designing QD-based pH-sensors. The most commonly explored sensors feature a change in the fluorescence intensity and/or 
wavelength in response to changes in the medium $\mathrm{pH}$.[36] A popular methodology for this is to decorate the QD surface with molecules that exhibit a pH-dependent optical profile (absorption and/or fluorescence), allowing modulation of QD emission by either energy or charge transfer interactions. For example, pH-sensitive fluorescent proteins (such as the mOrange) and dyes (such as the NIR carbocyanine dye, LS662) have been coupled to QDs, yielding pH-specific sensing platforms driven by energy transfer interactions.[86, 87] The use of $\mathrm{pH}$-sensitive fluorophores as FRET acceptors with QD donors was explored by two groups. Bao and coworkers immobilized several copies of the mOrgange protein on a QD to develop a FRET-based $\mathrm{pH}$-sensing platform. In particular, they tested the ability of their conjugates to report on $\mathrm{pH}$ changes in cell cultures.[86] Achilefu and co-workers employed QDs conjugated to the NIR carbocyanine dye as means of promoting $\mathrm{pH}$-dependent modulation of QD emission. They used both steady-state and time-resolved fluorescence measurements to show that such construct allows tracking of $\mathrm{pH}$ changes in buffer media from $\mathrm{pH} 2$ to $\mathrm{pH}$ 12.[87]

In one of the earlier works, Nocera, Bawendi and coworkers reported the use of QDsquaraine-dye conjugates to develop a FRET-based ratiometric pH sensor.[88] The QDs were encapsulated within an amphiphilic polymer, which was further chemically conjugated to a squaraine dye (Figure 4A). Because the absorption profile of the dye used changes with $\mathrm{pH}$, the spectral overlap between QD and squaraine dye also varies, resulting in a measured FRET efficiency that closely tracks changes in the medium $\mathrm{pH}$. In particular, under acidic conditions the dye exhibits higher absorption, which increases the spectral overlap integral and enhances the FRET efficiency; contribution of the FRET-sensitized dye emission is thus substantially increased. Conversely, at basic $\mathrm{pH}$ the absorbance of the dye is strongly reduced, leading to weaker spectral overlap and lower FRET efficiency. Here, the emission of the sample is dominated by the QD contribution. Therefore, tracking changes in the ratio of the QD and dye emissions provides a sensitive parameter reporting on $\mathrm{pH}$ changes in the medium.

In subsequent work, the authors developed an alternative design to achieve $\mathrm{pH}$ sensing, where control over energy transfer interactions relied on conformational change in QDoligonucleotide dye conjugates.[72] A chemically insensitive QD-dye pair was linked through a cytosine-rich oligonucleotide that undergoes a reversible conformational change (folding or 
unfolding) in response to changes in the $\mathrm{pH}$ of the surrounding medium (Figure 4B). Under basic conditions, the oligonucleotide adopts an extended duplex-overhang structure, which imposes a larger QD-to-dye separation distance. In acidic $\mathrm{pH}$ the protonated oligonucleotide forms a folded triplex motif, reducing the distance between the QD and dye. Due to the very high sensitivity of energy-transfer interactions to changes in the separation distance, conformational changes in the space linker lead to a substantial change in the measured fluorescence profile of the system (i.e., strong change in the relative contributions of the QD and dye to the measured PL spectrum with $\mathrm{pH}$ ). This conformational change produces a decrease in the measured FRET efficiency from $48 \%$ to $9 \%$ when the $\mathrm{pH}$ is switched from 6 to 8 . They showed that monitoring the emissions of both fluorophores can provide a ratiometric response, which can further be used to track changes in $\mathrm{H}^{+}$concentrations in solution and within endosomes of HeLa cells. [72]

Our group employed a slightly different approach, where $\mathrm{pH}$ sensing was based on tracking changes in QD PL due to charge transfer interactions between the QD and the redox active dopamine, as shown in Figure 4C. $[49,50]$ In an earlier study we self-assembled a Hispeptide labeled with a terminal dopamine onto the QD surface. We showed that immobilizing a rather large number of peptide-dopamine was required to measured large changes in $\mathrm{pH}$ induced QD PL loss. We further employed these conjugates to probe changes in intracellular $\mathrm{pH}$.[50] In subsequent studies we elaborated on the nature of the charge transfer interactions for this pair. We started with $\mathrm{pH}$-insensitive ZnS-overcoated CdSe QDs capped with a short poly(ethylene glycol)-appended dihydrolipoic acid (DHLA-PEG).[49] By introducing a fraction of amine-terminated ligands into the coating shell, we achieved control over the number of dopamine-isothiocyanate coupled to a nanocrystal. We found that strong quenching can be achieved using these constructs even with relatively low number of dopamine groups per QDconjugate. By combining the use of steady-state and time-resolved fluorescence along with transient absorption measurements, we were able to gain valuable information into the mechanism controlling the pH-dependent charge transfer interactions for this system. In particular, our combined studies showed that a QD simultaneously interacts with a mixture of catechol (i.e., reduced) and quinone (i.e., oxidized), two forms of the complex at equilibrium in the medium. When the $\mathrm{pH}$ of the medium is adjusted from acidic to alkaline conditions, there is 
a $\mathrm{pH}$-induced transformation of dopamine from catechol to quinone, along with lowering of the catechol oxidation potential. At $\mathrm{pH} 4$, the equilibrium favors a rather dominant concentration of catechol. When the $\mathrm{pH}$ of the medium becomes more basic (e.g., $\mathrm{pH} 10$ ), the equilibrium shifts towards a higher concentration of the oxidized quinone. These two forms of the complex interact differently with the QD: the reduced catechol becomes a better electron donor with increasing $\mathrm{pH}$, while quinone naturally provides an electron acceptor. Thus, when the $\mathrm{pH}$ is shifted from 4 to 10, a photoexcited QD can experience an electron transfer to the quinone while the easier to oxidize catechol can engage in electron transfer to the valence band of the photoexcited QD. This results in annihilation of the e-h pair, producing a pronounced loss in QD PL coupled with shortening of the luminescence lifetime.[49]

In a follow up study we found that this $\mathrm{pH}$-dependent quenching behavior was strongly dependent on the QD size (thus the energy levels) and separation distance between the QD and proximal dopamine groups.[51, 52, 89] We measured substantially larger quenching efficiencies, combined with more pronounced shortening of the carrier dynamics of these assemblies, for smaller size QDs and shorter PEG bridge. QD-dopamine conjugates prepared with tunable QD size and PEG bridges provide valuable platforms for the use in sensing and imaging, e.g., intracellular pH sensing.[50]

\subsection{Ion Sensing}

In vitro and in vivo sensing of several metal ions is a very active research field since many of those ions are ubiquitous and play critical roles in numerous intracellular signaling pathways. For example, $\mathrm{Ca}^{2+}$ ions are known to play a role in controlling cell metabolism, gene expression, vesicular trafficking and exocytosis.[90] Sodium and potassium ion channels rely on proton fluxes, driven by concentration gradients across the cell membranes to power a variety of active transmembrane processes, including neuronal signaling and cellular responses to external stimuli.[91-93] Cumulatively, this has driven the need for designing sensors that monitor the ion concentration within living cells, in order to understand the underlying principles of such processes. Sensing of heavy metal ions (e.g., $\mathrm{Hg}^{2+}, \mathrm{Pb}^{2+}$, and $\mathrm{Cd}^{2+}$ ) has also received great attention, due to the risks caused by the presence of such ions to human health and to the 
environment. Utilizing QD-based FRET or CT for signal transduction is one of the most promising approaches for detecting these ions in various media. Here, we elucidate this design using two representative examples.

In one example, Willner and co-workers reported on the use of thymine-rich nucleic acid-functionalized CdSe-ZnS QDs for the selective analysis of $\mathrm{Hg}^{2+}$ or $\mathrm{Ag}^{+}$ions. [94] The authors exploited the specific and selective recognition of $\mathrm{Hg}^{2+}$ and $\mathrm{Ag}^{+}$by thymine ( $\mathrm{T}$ )-rich- or Cytosine (C)-rich-DNAs, respectively. The ions form a bridge with these bases and transform the oligonucleotide from single to double strand (hairpin-like) conformation, with the ions trapped within the DNA double strands. This brings the metal ions close to the QD surface, promoting charge transfer interactions with photoexcited QDs and quenching of their emission. The kinetic study showed that when mixed with a solution containing $0.1 \mathrm{mM} \mathrm{Hg}^{2+}$, the QD-oligonucleotide conjugates experience 85\% PL loss (compared to control dispersion without any Hg ions) after 24 min incubation. A similar "turn-off" response was obtained in the presence of silver ions if the thymine-rich nucleic acids were replaced by cytosine-rich sequence. The detection limit using these probes could be as low as $10 \mathrm{nM}$ for $\mathrm{Hg}$ ions and $1 \mu \mathrm{M}$ for $\mathrm{Ag}$ ions.

In the second example, Feltz and co-workers designed a FRET-based sensor targeting calcium ions using CaRuby, a red-emitting calcium indicator, as the acceptor (Figure 5).[90] The nanocrystals were surface-functionalized with a mixture of cysteine-rich phytochelatins presenting either a terminal thiol or amine; the cysteine groups promote coordination of the peptides onto the QDs. CaRuby was then coupled to the QD surface using thiol/maleimide cross-coupling. The red-emitting CaRuby is an extended Rhodamine linked to a $\mathrm{Ca}^{2+}$ chelating BAPTA moiety. Alone this complex is a dark quencher, but chelation of $\mathrm{Ca}^{2+}$ onto the BAPTA group (e.g., in a $\mathrm{Ca}^{2+}$ rich media) alters the BAPTA conformation and results in PL emission of this compound. When QD-CaRuby conjugates were dispersed in $2 \mathrm{mM} \mathrm{Ca}^{2+}$ solution, they measured strong QD quenching with a FRET efficiency that increased with the number of CaRuby per QD, as expected for one donor-multi-acceptor system, along with a FRET-sensitized increase in the dye emission. Control experiments using similar concentration of $\mathrm{Ca}^{2+}$ mixed with CaRuby dye alone (no QDs) yielded much smaller dye emission, due to the absence of FRET interaction with the QDs. To demonstrate the $\mathrm{Ca}^{2+}$ sensing ability of the QD-conjugates inside 
live cells, the nanocrystals were further conjugated with a small cell penetrating peptide (CPP) to facilitate their intracellular delivery. The conjugates were incubated with HEK293 cells expressing N-methyl-D-aspartate receptors (NMDARs), which are cation-permeable channels mediating $\mathrm{Ca}^{2+}$ influx. Cells pretreated with QD-CaRuby conjugates were suspended in a buffer medium containing $10 \mathrm{mM} \mathrm{Ca}^{2+}$. Addition of NMDAR agonists (glutamate and glycine) to the cell suspension resulted in an increase in CaRuby fluorescence. This is attributed to the NMDARmediated $\mathrm{Ca}^{2+}$ cross-membrane entry, confirming the responsiveness of the QD-biosensors inside cells. Pre-incubation of the cells with APV, a NMDAR antagonist, did not produce similar CaRuby emission, presumably due to the absence of intracellular $\mathrm{Ca}^{2+}$ (see Figure 5).

\subsection{Sensing of Bio-molecular Interactions}

4.3.1 Competitive sensing using QD-protein/antibody conjugates. Several studies have tested the effectiveness of using QD-protein or QD-antibody-fragment conjugates as sensors for the detection of small molecule analytes.[78, 95] In a competitive sensing format, a dye-labeled analog is first captured by the QD-protein/antibody conjugate, resulting in FRET-induced quenching of the QD emission. When the target molecules are added to the solution, they displace the dye-labeled analog away from QD surface, producing a progressive recovery of the QD emission, due to reduction in FRET interactions. Analyzing the time- and concentrationdependent QD PL recovery provides information about the sensing kinetics and estimates of the binding constant.

Earlier examples of such sensing configuration include the detection of sugar maltose using QDs conjugated to the maltose binding protein (QD-MBP conjugates).[78] A similar design also developed by our group utilized QD-antibody-fragment conjugates to assemble a sensor that is specific for the detection of the explosive 2, 4, 6-trinitrotoluence (TNT) in aqueous solutions (Figure 6A).[95] The sensor was constructed by attaching His-tagged anti-TNT antibody fragments on QDs via metal-histidine affinity coordination. An analog made of a dark quencher labeled to TNB (BHQ-10-TNB), prebound to the antibody quenched the QD emission due to FRET interactions with the QDs (Figure 6A). Addition of soluble TNT displaced the BHQ-10-TNB and reduced FRET to baseline interactions, resulting in a TNT concentration-dependent recovery 
of the QD emission.

4.3.2 Sensing enzymatic activity using QD-peptide conjugates. Designing QD-based sensors targeting enzymatic activity has generated great interest in the community, and several groups have applied QD-conjugate to monitor enzymatic activity mostly in solution conditions. The enzyme proteases, for instance, catalyze the breaking of specific peptide bonds in proteins. They are heavily involved in many normal biological processes as well as in diseases, including cancer, stroke and infection.[96-98] A variety of FRET-based sensors have been designed to monitor the activity of proteases by detecting changes in the FRET efficiency between two proximal fluorophores attached to the ends of the peptide substrate.[99, 100] A commonly utilized configuration with QDs consists of a QD conjugated to a few dye-labeled peptidesubstrates (containing a cleavage sequence).[101-106] Conjugation onto the QD produces a valence-dependent quenching of the PL via FRET. When the target enzyme is added, it specifically cleaves the recognition sequence, removing the dye away from the QD surface. This results in progressive recovery of the QD signal. Monitoring the QD PL change with enzyme concentration and time provides information on the proteolytic activity.[105-109]

Several QD-peptide substrate constructs were developed by a few groups. In one of the earlier examples the dye-labeled peptide used had a sequence that can be cleaved by either trypsin or collagenase.[110] Following mixing of the QD-peptide-dye conjugates with the enzyme, recovery of the QD emission (which was found to vary with enzyme concentration and incubation time) was utilized to provide information on the rate of enzymatic reaction. In addition, the authors showed that in the presence of an inhibitor, the rate of QD PL recovery was substantially smaller, due to a reduction in protease activity. In the second example, we have developed a series of tailored QD-peptide conjugates containing sequences specifically recognized by the proteases caspase-1, thrombin, collagenase and chymotrypsin.[111] The modular peptides contained an N-terminal polyhistidine domain for self-assembly on the QDs and a C-terminal cysteine-thiol for labeling with a dye. We formed four sensing assemblies and tested them against the four different proteases above. The relative changes in FRET efficiency measured in the presence of the target enzyme, at a given concentration, were analyzed to determine the kinetic parameters (namely, the Michaelis constant, $\mathrm{K}_{\mathrm{M}}$, and the enzymatic 
maximum velocity, $\left.V_{\max }\right)$. Additional data collected in the presence of inhibitors allowed identification of the mechanism of inhibition, i.e., competitive vs. non-competitive.

Subsequent studies followed similar experimental design and rationale to screen for other enzymes. For example, Stevens and co-workers tested their QD-peptide conjugates to study the activities of the tyrosine kinases Abl and Src, see Figure 6B.[108] Mercaptopropionic acid-modified QDs were conjugated to peptide substrates by metal-histidine driven selfassembly as done above. The conjugates were incubated with tyrosine kinase in the presence of excess ATP. The generated phosphorylated peptides were then detected by adding a phophotyrosin-specific monoclonal antibody labeled with a dye (Figure 6B). Steady-state emission spectra revealed a progressive decrease in QD emission concomitant with an increase in dye signal as a function of enzyme concentration; FRET quenching efficiency as high as $75 \%$ was measured. In the absence of ATP or when an inactive peptide substrate was used, no FRET interactions were measured, confirming that the measured changes in the fluorescence signature of the samples were originating from ATP-dependent tyrosine phosphorylation. In comparison, when the enzyme was preincubated with a kinase inhibitor (staurosporine), prior to the addition of ATP, the measured fluorescence intensity ratio was very different from the above results, which reflects the effects of staurosporine inhibition of enzymatic activity. Subsequently, they expanded this design and used two color QD-peptide conjugates to demonstrate multiplex sensing, where the activities of two distinct enzymes have been probed.[105] A recent study by Wang and co-workers reported the design of a FRET-based QD sensor targeting the enzyme matrix metalloproteinase (MT1-MMP, an important indicator of cancer) both in solution and on cell membranes.[112] One of the key features of the sensor design detailed in this study is the use of a peptide substrate (AHLR, cleavable by MT1-MMP) that is inserted between a cationic and an anionic sequences, along with a C-terminus Cy3 dye and a N-terminus His6 tag for self-assembly on CdSe-ZnS QDs; the peptide structure further included a RGD sequence. The peptide forms a bent structure, weakly-stabilized by electrostatic attractions between the cationic and anionic sequences (Figure 6C). Once self-assembled on the QDs, the bent sequence imposes a configuration where the Cy3 is very close to the QD surface, resulting in high FRET quenching of the QDs, or a weak FRET ratio defined as the ratio 
of QD PL to Cy3 PL. They then tested the capacity of this construct to sense the activity of the active catalytic domain of MT1-MMP (i.e., MT1-CAT) in solution and showed that indeed incubating the QD-petide-Cy3 conjugates with MT1-CAT resulted in large changes in the FRET ratio (as high as 5). This was attributed to cleavage of the AHLR sequence, separating of the two charged domains and displacing the Cy3 away from the QD surface. Changes were found to strongly depend on the enzyme concentration and incubation time. They also tested this sensor in a human cancer cell line (MDA) expressed with high MT1-MMP activity; the cells were incubated with the QD-conjugates and imaged using fluorescence microscopy. Here too they measured an increase in the FRET ratio combined with a more pronounced endocytosis of the QDs. This was attributed to the specific binding of the sensor to the integrin in cell membrane, promoting cleavage of the peptide by the active MT1-MMP. Additionally, cleavage of the peptide sequence exposed the arginine-rich domain, which promoted intracellular uptake of the QD-conjugate with high QD emission (dye-free system). In control experiments they found that when an MT1-MMP-specific inhibitor was added to the culture, marginal changes in the FRET ratio combined with minimal intracellular uptake were measured. These results confirm that the sensor also specifically interacts with the membrane bound enzyme.[112]

4.3.3 Detection of DNA hybridization using QD-oligonucleotide conjugates. Here too the QDs act as both energy donor and scaffold for the immobilization of oligonucleotide sequences. The hybridization process is usually detected by probing FRET interactions between the QD donor and a complementary probe sequence labeled with a dye (acceptor). This includes a single sandwich made of two complementary sequences, or a more complex sandwich made of three DNA strands: one target partially hybridized with two complementary sequences. An alternative strategy is to use molecular beacon structures, where hybridization with a target sequence modulates the separation distance of the integrated donor-acceptor assemblies (i.e., QD-DNA beacons).

Earlier FRET-based sandwich assemblies as sensors targeting nucleic acid that use QDs have been demonstrated by Wang and colleagues.[113] In their design each DNA nanosensor consisted of two specific oligonucleotide probes, which included a reporter probe labeled with a Cy5 dye and a capture probe labeled with biotin, in addition to a streptavidin-modified QD. 
When a target sequence was introduced in the solution, a sandwich structure was formed on the QD; here the biotinylated probe is complementary to half of the target sequence, while the Cy5-labeled probe is complementary to the other half of the same target (Figure 7A). Following incubation the biotin-modified sandwich assemblies bound onto the QD surface, producing changes in the fluorescence emission as anticipated for FRET interactions between the QD and attached dye; this accounts for the presence of the target sequence in the medium.

Willner and coworkers have expanded the general FRET-based molecular beacon to the use of $\mathrm{CT}$ interactions as a means of altering QD emission and for detecting hybridization interactions.[114] They immobilized hairpin structures on the QD surfaces. The hairpin DNA included a recognition sequence for the target sequence and a G-quadruplex sequence, in a cage configuration (in the stem region), as illustrated in Figure 7B. In the presence of the target, the hairpin structure opened, leading to the self-assembly of Fe(III)-protoporphyrin IX (hemin) with G-quandrulex. The hemin/G-quandrulex exhibits a quasi-reversible redox properties that correspond to the $\mathrm{Fe}(\mathrm{III}) / \mathrm{Fe}(\mathrm{II})$-protoporphyrin IX couple. The redox state of the hemin complex lays between the energy levels of the QDs, which facilitates the transfer of conduction band electrons (in photoexcited QDs) to the hemin complex. As the surface coverage of QDs by the hemin/G-quandrulex quencher units was controlled by the concentration of the target probe, the fluorescence quenching of QDs provided a quantitative readout for the abundance of the target molecules.

In a recent report, Dubertret and co-workers have reported a more efficient and better controlled coupling of single stand DNA (ssDNA) onto a QD via thiol maleimide coupling.[115] Here they started with a polymer coating that presents a few lipoic acid anchors along with zwitterionic moieties to promote hydrophilicity. They engineered the polymer coating of the QDs such that several thiol groups were made available on the QDs following ligation. Maleimide-modified ssDNA sequences were prepared by reacting amine-terminated ssDNA with a Sulfo-SMCC cross-linker (Sulfosuccinimidyl-4-[N-maleimidomethyl]cyclohexane-1carboxylate). This allowed application of the more efficient maleimide-to-sulfhydryl coupling to attach a controlled number of ssDNA onto well-stabilized thiol-presenting QDs. They then tested the biological activity of the QD-ssDNA conjugates by reacting it with biotinylated 
complementary ssDNA sequence, followed by incubation with streptavidin-coated agarose beads. They showed that indeed formation of fluorescent agarose-QD structures could be achieved only if the biotin-ssDNA were complementary to the sequence bound to the QDs. Conversely, configurations where QDs only, or QD-ssDNA preincubated with noncomplementary biotin-ssDNA sequence, were mixed with the streptavidin agarose beads, yielded non fluorescent samples.[115] The combination of multi-coordinating zwitterion polymer coating and use of thiol-to-maleimide reaction seems to provide QD-DNA conjugates with controlled valence and colloidally stable. These QD-sSDNA conjugates can potentially enhance the utility of QD in hybridization assays and gene detection.

\subsection{QD-Metal Complex Conjugates and Photodynamic Therapy}

Energy and/or charge transfer interactions between QDs and metal complexes have been used by researchers to develop applications in molecular catalysis, water splitting, and solar energy conversion.[116-120] Additionally, the use of QD-based FRET to enhance the efficiency of photodynamic therapy (PDT) agents to treat cancer has received great attention.[121-125] PDT is a process that involves the transfer of excitation energy from a photosensitizing agent to a nearby oxygen molecule, resulting in the formation of reactive singlet oxygen.[124, 125] Being highly reactive species with short diffusion length (less than $0.1 \mu \mathrm{m}$ ), singlet oxygen initiates cytotoxic reactions in cells and tissues, which makes PDT useful for potential treatment of cancerous cells and tissues. The use of water-soluble and surface-functionalized QDs is attractive because the nanocrystals serve as scaffolds for immobilizing several PDT active compounds together with targeting molecules. This allows not only improving the solubility of the PDT compounds but also promotes homing onto the targeted tumor. In addition, due to their large extinction coefficients, the QDs can act as efficient energy transducers for enhancing photoexcitation of PDT compounds through FRET interactions. Moreover, the QDs have high two-photon cross section, which allows potential excitation of the system using NIR (near infrared) irradiation for deep-tissue penetration, a feature that most PDT molecules cannot fulfill.[122]

In one of the earlier works introducing QDs as a potential enhancer of PDT signal, Burda 
and coworkers demonstrated the FRET-based production of ${ }^{1} \mathrm{O}_{2}$ using QD-silicon phthalocyanine (Pc4) hybrid system.[121] Due to the strong absorbance at long wavelengths (672 nm) and high ${ }^{1} \mathrm{O}_{2}$ efficiency (43\% under direct photoactivation), Pc4 is ideal for tissue penetration with minimal cutaneous photosensitivity. To form the conjugation, Pc4 was attached to the QD through an alkyl amino group on the photosensitizer's axial substituent. Upon excitation at 488 $\mathrm{nm}$, the QD acted as the energy donor to Pc4, while Pc4 acted as "FRET bridge" to excite the proximal oxygen. The combination of semiconductor QDs and PDT photosensitizers enabled the use of an excitation wavelength where the photosensitizers alone do not absorb. This provided the flexibility of using variable excitation wavelengths to activate the sensitizer molecules. However, integration of the QD-Pc4 complex within biological systems has been rather limited, due to a combination of poor water solubility and the use of non-covalent conjugation.[122] Following this investigation, many biocompatible covalent systems composed of QD and various chromophores, such as porphyrins, phthalocyanines, inorganic complexes and organic dyes as energy acceptors, have been developed for singlet oxygen production. Weiss and co-workers have developed hydrophilic QD-photosensitizer conjugates using Rose Bengal and Chlorin e6.[122] This was achieved by attaching the PDT molecules to phytochelatin-related peptides (thiol-rich) via EDC/NHS coupling, followed by subsequent cap exchanged on the QDs. They compared the generation of singlet oxygen achieved via either indirect excitation (through FRET from the nanocrystals), or via direct excitation of the photosensitizers. They also explored the effects of excitation wavelength, nature of the sensitizer used (Rose Bengal vs Chlorin e6), as well as the valence of the QD-PDT complex. They, for example, achieved singlet oxygen generation quantum yield, through indirect FRET excitation, as high as 0.31 using red-emitting QDs coupled to Chlorin e6 with 532-nm excitation.

Beyond singlet oxygen generation, QDs can serve as potential FRET antennas for other photosensitized reactions of metal complexes in therapeutic applications. For example, nitric oxide (NO) is an endogenous bioregulator that plays important roles in vasorelaxation, immune response, and tumor growth and suppression, and may increase the effectiveness of $\gamma$-radiation treatment of tumor tissue.[126] Biologically active NO molecules can be generated in situ from photochemical nitrosyl and nitrito complexes. Ford and co-workers tested a promising 
photochemical NO precursor, chromium complex trans- $\mathrm{Cr}(\mathrm{cyclam})(\mathrm{ONO}) 2^{+}(\mathrm{CrONO})$ (see Figure 8).[127] Although CrONO is an efficient photogenerator of NO (NO $=25 \%$ ), the practical application is limited by its poor absorption cross-section, especially at long visible wavelengths. To circumvent this spectroscopic limitation, the authors used luminescent QDs as energy antennas to sensitize CrONO molecules adsorbed on the surface of the nanocrystals by electrostatic interactions. This structure allowed taking advantage of the large single- and twophoton absorption cross-sections and large surface-to-volume ratio of the QDs, to achieve efficient excitation in the visible and NIR; it also allowed the assembly of several CrONO groups per QD-complex conjugate. They indeed found that photoexcited QDs transferred energy to the quartet-excited state of CrONO, via FRET interactions, followed by release of NO as a result of internal conversion to the reactive doublet state. Enhancements in NO production were $~ 6$-fold higher than that measured for CrONO in the absence of QDs. Due to the large two-photon absorption cross-sections of QDs, this sample configuration is promising for harvesting NIR excitation light and can be beneficial for use in biological matrices such as cell cultures and tissues.

\section{Interactions of QDs with Metal and Metal Oxide Nanoparticles and Graphene}

In all the biosensing configurations discussed above, QDs engages in energy transfer or charge transfer interactions, and it has been paired with various dye acceptors, redox active molecules, and metal ions. Metallic nanostructures, in particular gold nanoparticles, have been explored as highly effective quenchers of QD and dye emissions alike, due to a process that is more effective than the Förster dipole-dipole interactions mechanism. Instead, interactions with metal nanostructures have been discussed within the framework surface metal quenching, which is shown to extend over much larger separation distances beyond those allowed by FRET.[128, 129] In addition to metallic nanoparticles, metal oxide and carbon nanomaterials have also been paired with QDs for energy and/or charge transfer interactions. Herein, we discuss a few representative examples where such materials have been paired with QDs, to probe energy and/or charge transfer interactions.

\subsection{Interactions with Gold Nanoparticles}


The use of gold nanoparticle quenchers has attracted much attention, because of the wealth of core materials that can be prepared with varying size, shape, stoichiometry and surface properties.[130-133] Wu and co-workers explored the effects of changing the Au nanoparticle (AuNP) size on the PL quenching of QDs (Figure 9A).[134] In this study, three different-size nanoparticles $(3,15$, and $80 \mathrm{~nm}$ in diameter) with distinct surface plasmon resonance (SPR) features were used. The AuNPs were linked to the QDs through a rigid double-stranded DNA spacer, and the separation distance was controlled by varying the length of DNA sequences used. They found that for the $3 \mathrm{~nm}$ AuNPs which have negligible SPR absorption band, the QD fluorescence quenching efficiency exhibited a weaker dependence on the separation distance (i.e., quenching efficiency varied as $1 / r^{4}$ ), indicating that the interactions and quenching are governed by nanometal surface energy transfer (NSET) mechanism.[129, 135] Conversely, when the 15 and $80 \mathrm{~nm}$ nanoparticles which have strong SPR absorption bands that overlap with the emission profile of the QDs were used, the quenching efficiency exhibited $1 / r^{6}$ dependence, suggesting that interactions are dominated by a dipole-dipole coupling associated with the more conventional FRET. Furthermore, they measured higher quenching efficiency for the 80 $\mathrm{nm}$ nanoparticles compared to the $15 \mathrm{~nm}$ and $3 \mathrm{~nm}$ nanoparticles, which was attributed to a larger spectral overlap between the SPR absorption and QD emission spectra. However, it has been found in other studies that the data on the quenching of QD emission by proximal AuNPs is consistent with the NSET model.[132, 133] Although several aspects of the PL quenching in QD-AuNP systems remain unclear, the NSET model seems to provide better account for the data measured for the smaller to medium size gold NPs, as high quenching efficiencies have been consistently measured when AuNPs were used compared to conventional D-A dye pairs.

We should note that there are instances where authors have reported that instead of PL quenching proximity to $\mathrm{Au}$ and $\mathrm{Ag}$ nanostructures also results in PL enhancement of dyes and QDs alike.[136, 137] Enhancement in the PL emission of QDs, for example, has been mostly explored using layered structures of QDs and AuNPs,[138] QDs spatially arrayed around Au nanorods (AuNRs),[136] and QDs arrayed around large scale nanoprisms.[139] Enhancements are observed at larger separation distance where energy transfer quenching is weaker, and they are often much easier probed using single molecule spectroscopy.[140] Such effects are also 
easier to observe when the starting fluorophores exhibit rather weak quantum yield. It has been less commonly observed for structures involving AuNPs in solution phase.[141-143] This phenomenon has been attributed to the interactions of the dye/QD exciton with the strong electric field generated near plasmonic hots spots (e.g., near edges and between two AuNRs).

\subsection{Charge Transfer Interactions of QDs with Titanium Dioxide Nanostructures}

Titanium dioxide $\left(\mathrm{TiO}_{2}\right)$ nanostructures have emerged as one of the most useful materials in practical applications ranging from pharmaceuticals, photocatalysis to solar cells.[144] Photosensitization of $\mathrm{TiO}_{2}$ nanostructures with semiconductor quantum dots, via charge transfer interactions, has been demonstrated in photocatalysis and in the development of fully inorganic solar cells.[30, 31, 33, 145] For example, Kamat and coworkers probed the sizedependent rate of charge transfer from excited CdSe QDs to $\mathrm{TiO}_{2}$ nanoparticles in close-packed films (Figure 9B).[69] They utilized mercaptopropionic acid linkers and exploited the preferential binding affinity of thiol groups to QDs and of carboxylic groups to the surface of $\mathrm{TiO}_{2}$, to form the charge transfer hybrid structure. Five different sizes of QDs ranging from $2.4 \mathrm{~nm}$ to $7.5 \mathrm{~nm}$, with first excitonic $\left(1 \mathrm{~S}_{3 / 2} 1 \mathrm{~S}_{\mathrm{e}}\right)$ peak corresponding to emission ranging from $509 \mathrm{~nm}(2.44 \mathrm{eV})$ to $645 \mathrm{~nm}(1.92 \mathrm{eV})$ were prepared and tested. Controlling the nanocrystal size provided a simple way to tune the band gap and energy levels of the charge carriers. They found that the rate of charge transfer from excited $\mathrm{QDs}$ to $\mathrm{TiO}_{2}$ nanoparticles, extracted using femtosecond transient absorption measurements, increased with decreasing size. Furthermore, they showed that bleach of the band edge absorption was dominated by the presence of the electrons in the conduction band, with negligible contribution from the holes. This result was attributed to the more favorable conduction band energies for injecting electrons into $\mathrm{TiO}_{2}$. As the nanocrystal size decreased, an enhanced recovery of transient bleach was measured, indicating the faster electron transfer rates for smaller $\mathrm{QDs}$ to $\mathrm{TiO}_{2}$ in the sample.

\subsection{Nanocarbon-based Quenching of QD Emission}

Currently, hybrid systems consisting of carbon-based materials, such as carbon nanotubes (CNTs), graphite and graphene have generated great interests.[146-151] Graphene, in particular, exhibits promising electronic properties such as very high carrier mobility.[152] Combining 
them with QDs can provide hybrid nanomaterials that exhibit unique properties with potential applications in light-harvesting and photovoltaic devices. For example, Brus and co-workers probed energy transfer interactions between QDs and proximal single- or few-layers of graphene at the single molecule level.[148] They measured strong fluorescence quenching for QDs deposited on graphene sheets (see Figure 9B), and the PL quenching was strongly dependent on the number of graphene layers used. They further showed that the kinetics associated with resonant energy transfer were faster than those measured for electron transfer from CdSe-ZnS nanocrystals to surface-adsorbed graphene. This suggests that QD quenching is due to FRET, not electron transfer interactions.

One interesting aspect of these hybrid assemblies is the change in the PL blinking kinetics measured for single QDs, when they are paired with carbon-based materials. For example, Brus and coworkers measured long "off" periods, occurring when single QDs were deposited on a quartz substrate, but not when the QDs were deposited on graphene materials (Figure 9C).[148] This property was attributed to the fast and very efficient energy transfer from the QD to graphene, which effectively competes with the Auger ionization process widely used to explain the sustained blinking in single QD emission. Within this Auger recombination model, blinking is attributed to charge carrier trapping (following excitation and carrier generation) at the surface of the nanocrystals or in the surrounding matrix, leading to a nonradiative exciton recombination and suppression of emission.[153, 154] This process is stochastic leading to an alternation between "on" and "off" emission intervals under continuous laser excitation. The reduction of the "off" times, measured in the presence of the carbon layer, indicates the presence of energy transfer from the nanocrystal to the carbon layer. Weller and co-workers also investigated the blinking behavior of CdSe-CdS nanorods when interacting with a carbon layer through a separation barrier (with thickness between 9 and $160 \mathrm{~nm}$ ). They found that PL quenching of the QDs (probed at the single molecule level) was strongly dependent on the separation distance from the carbon film.[150] Furthermore, they found that the faster energy transfer measured for the structures with smaller separation distance, while drastically reducing the PL intensity, has also altered the blinking behavior of single QDs. A nearly continuous emission and zero "off" occurrence in the measured time-trace patterns was recorded, in 
agreement with the observation reported by Brus and co-workers.[148]

\section{Configurations using QDs as Energy Transfer Acceptors}

Use of QDs in energy transfer studies and sensor design has mostly exploited them as FRET donors. This was illustrated in the literature and by the number of examples described above. In contrast, utilizing QDs as energy transfer acceptors has been rather limited, due to a few photophysical factors. One practical constraint results from the fact that, in common energy transfer configurations, QDs are more efficiently excited than most dyes and fluorescent proteins, because they have broad absorption and large extinction coefficients ( $10-100$ times than that of conventional dyes). This makes isolating the contribution to the QD emission due to FRET interactions with a donor fluorophore rather complicated. Moreover, the PL lifetimes of QDs are larger than those of dyes (10-20 ns compared to 1-5 ns for dyes), and this makes timeresolved fluorescence measurements difficult to analyze. In one early study testing FRET from organic dyes to CdSe-ZnS QDs we found that no measurable FRET could be detected via either steady-state or time-resolved fluorescence measurements.[155] FRET between different size QDs has, however, been observed in close-packed films using both steady-state and timeresolved fluorescence measurements and data were interpreted within the Förster formalism.[156, 157]

To circumvent these complications, rare earth fluorophores, such as Tb-chelates with very long excitation lifetimes ( ms), have been employed by Hildebrandt and coworkers to implement energy transfer to QD acceptors. They combined this with the use of time-gated fluorescence measurements, thereby isolating the QD signal resulting from FRET interactions to time scales far exceeding their natural lifetimes.[158] They explored several optimization conditions which allowed them to outline a method for data analysis by reporting the interaction efficiencies using FRET ratio (QD-PL/Lanthanide-PL) instead of the more conventionally used FRET efficiency defined in equations 5 and 13. In some of their earlier studies they probed FRET interactions using biotin-sptreptavidin binding between biotin-QDs and streptavidin-labeled Tb-complexes.[159] In subsequent work they explored the capacity to use the $Q D$ as dual platforms acting as an energy acceptor to Tb-complex and donors to 
AlexaFluor 646 (A647).[160] In a more recent study they showed that combining Tb-chelate donors with QD acceptors they could achieve multiplexing detection of several microRNAs (miRNAs) in a small-volume buffer or serum-based sample, as schematically summarized in Figure 10A.[161] The detection of the target miRNA is specific as it occurs only when the oligonucleotide double strand structure is formed on the QD, bringing the Tb-complexes close to the nanocrystal surface.

A more suitable configuration exploits the natural ability to generate fluorescence signal from donor molecules without relying on optical excitation, using chemi-luminescent and bioluminescent compounds, instead of conventional fluorophores. Here the donor excitation comes from an enzyme-catalyzed biological reaction. This has been referred to as selfillumination of the QDs via bio-luminescence resonance energy transfer (BRET) or chemiluminescence resonance energy transfer (CRET). It involves the nonradiative transfer of excitation energy from excited molecules to the ground state QD acceptor. Thus, BRET and CRET are analogous to FRET, but the donor energy comes from a chemical reaction rather than direct external excitation. This self-illuminating configuration is promising for imaging opaque biological samples where background endogenous auto-fluorescence could be eliminated in the absence of any external photoexcitation. Below we illustrate a few representative examples where BRET or CRET excitation of QD acceptors has been demonstrated and further applied for sensor development.

One of the early studies exploring BRET was reported by Rao and coworkers, where they assembled several copies of a light-emitting protein, Renilla reniformis luciferase, to a single QD.[162] They selected an 8-mutation variant of luciferase (Luc8) with improved catalytic efficiency, as bioluminescent donor.[162] An average of 6 Luc8 proteins was coupled to $\mathrm{COOH}$ modified red-emitting QDs. They measured a strong signal centered at $655 \mathrm{~nm}$ (QD peak) upon the addition of coelenterazine substrate; this signal is superposed onto the one characteristic of luciferase (see Figure 10B). The QD contribution was attributed to nonradiative transfer of excitation energy to the central QD (i.e., BRET-sensitized emission), since direct excitation of the QD has not taken place. To quantify this interaction and extract a measure for the energy transfer efficiency of this process, the authors defined a BRET ratio between the QD $(A)$ and the 
Luc8 (D) emissions, similar to the FRET ratio used above with Tb-QD pairs. They measured a BRET ratio that depended on the 'spectral overlap' between Lu8 emission and QD absorption and on changes in D-A separation distance; closer proximity provided higher BRET ratios. Additionally, they found that by coupling the same enzyme simultaneously to QDs with distinct emissions ranging between $605 \mathrm{~nm}$ an $800 \mathrm{~nm}$, composite emissions from all QDs could be measured when the reaction was initiated (i.e., multiplex BRET). They further tested these conjugates in vivo within mice tissues. In particular, addition of the substrate to cell cultures loaded with QD-Luc8 conjugates produced composite luminescence spectra characteristic of the QD combination used. High signal-to-background BRET ratios could also be measured when performing in vivo imaging in mice even with small amounts of QD-Luc8 conjugates. In a follow up study, they applied this concept to develop a BRET-based QD biosensor for detecting the activity of matrix metalloproteinase-2 (MMP-2).[163]

Willner and co-workers showed that chemi-luminescence can also be used to sensitize QD emission in the absence of external photoexcitation.[164] They used the hemin/Gquadruplex horseradish peroxidase (HPR) (a structure mimicking DNAzyme), which acts as catalyst to generate a chemi-luminescent signal through the oxidation of luminol by $\mathrm{H}_{2} \mathrm{O}_{2}$. They started with two DNA sequences, (5) and (6), which include four distinct domains: domains I and II provide the HRP-mimicking DNAzyme while domains $\mathrm{V}$ and VI specifically bind to the target ATP (i.e., the latter binding domains constitute an "anti-ATP" aptamer, as shown in Figure 10C). The presence ATP provides the necessary additional stability for the active hemin/Gquadruplex, by interacting with the domains $\mathrm{V}$ and $\mathrm{VI}$ in the oligonucleotide sequences. They found that the assembled DNAzyme structure catalyzed the $\mathrm{H}_{2} \mathrm{O}_{2}$ oxidation of luminol, generating chemi-luminescence signal at $\lambda=420 \mathrm{~nm}$. Assembly of this structure on a CdSe-ZnS QD was achieved by introducing a terminal thiol on sequence (6), allowing the formation of QDDNAzyme/ATP/hemin/G-quadruplex conjugates (Figure 10C). In the presence of $\mathrm{H}_{2} \mathrm{O}_{2}$ and luminol this assembly produced a measurable QD signal, attributed to nonradiative energy transfer of the chemi-luminescent signal from the catalyzed reaction of luminol by the caged hemin/G-quadruplex, CRET, to the central QD. The structure essentially provided an internal light source to sensitize the emission of the linked QDs. The QD signal was found to depend on 
the concentration of ATP used. The measured QD emission, thus CRET efficiency, increased at higher ATP concentration; the latter improve the number and stability of the hemin/Gquadruplex structures, yielding higher chemi-luminescence signal for more efficient sensitization of the QDs. The same concept using different oligonucleotide sequences was applied to detect the presence of $\mathrm{Hg}^{2+}$ ions via CRET.[164]

\section{Importance of the Charge and Energy Transfer in QD-based Electronic Devices}

This section will be rather limited in scope. We simply would like to show that charge transfer processes involving QDs are not only limited to configurations where the nanocrystals interacts with redox complexes as a means of controlling the QD signal. Indeed, charge transfer and charge separation are very important properties that can affect and control the operation and efficiency of several optical and electric devices. For example, the operational efficiency of lightemitting device depends on: the structure and efficiency of the charge injection into the hole and electron transport layers, the mobility across the layers in the hetero-structure, and the formation and recombination of excitons. Similarly, charge separation and carrier diffusion following photon absorption are critically important to the operation of photovoltaic cells. There have been already several recent review articles critically evaluating the structures, operating mechanisms and challenges for designing optical and electric devices.[11, 31-35] Here, we discuss a few examples demonstrating the importance of charge and/or energy transfer (in a more conventional transport context) and its relation to energy mismatch, and how these properties play a critical role in QD-based light-emitting and solar cell devices.

Colloidal quantum dots are greatly promising for developing high-efficiency photovoltaics based on low-cost materials.[30, 32-35] The combination of bright, size-tunable emission and ease of processing using solution phase conditions have motivated the use of QDs in high-quality display and solar energy conversion applications. Recent advances have brought the external quantum efficiency of QD light-emitting devices (QD-LEDs) to 20\% and the power conversion efficiency of QD solar cell to $9 \% .[165,166]$ Most routes to higher efficiency of photovoltaics undoubtedly require a better understanding of the operating mechanisms in these devices.[34] 
QD Vision Inc. has reported in 2013 the fabrication of hybrid multilayer red-emitting LEDs using an inverted organic-inorganic hybrid device structure based on CdSe-CdS QD emitters.[167] They used a multi-layer device fabricated by spin-coating an electron transport layer of ZnO nanocrystals, a QD emitting layer, followed by deposition via thermal-evaporation of a hole transport layer (made of small molecule spiro-2NPB) and a hole injection layer (made of LG-101); the structure and energy diagram are shown in Figure 11A. The strong coupling between the QDs and the adjacent layer of ZnO nanocrystals facilitates electron transfer, and is responsible for both injection and maintenance of an optimal charge balance in the QD emitting layer. The authors also found that the QD-LED performance could be modified by controlling the location of the exciton recombination zone within the quantum dot layer with respect to the interface with the ZnO layer. Using these conditions, the highest electroluminescence efficiency was achieved using a device with 45-nm thick QD layer, with an external quantum efficiency of $\sim 18 \%$.

Using similar inverted hybrid device structure, full-color (red, green and blue, RGB) QDLEDs have been developed by Lee and coworkers (Figure 11B).[168] Although the external quantum efficiencies were relatively low, the brightness was comparable to phosphorescent organic light-emitting diodes. They found that the device performance was also greatly affected by the hole and electron transport layers (e.g., electron transport layer made of ZnO nanocrystals). For instance, QD-LEDs employing hole transport layer with lower HOMO energy levels showed higher external quantum efficiency, which essentially indicates that the selected hole transport materials should have low enough energy to allow hole injection into the QD valence band. More recently, Peng and co-workers showed that by inserting an insulating PMMA layer $(6 \mathrm{~nm})$ between the QD and the ZnO layers, an efficiency of $\sim 20 \%$ could be achieved for the red-emitting QD-LEDs (Figure 11C).[166] The excess electron injection in QD emissive layer is known to affect the charge balance, leading to degradation in device performance. Thus the incorporation of a thin PMMA layer modified the QD/ZnO interfacial interaction and optimized the charge balance in the device, thereby improving the device efficiency. Additionally, devices containing a PMMA layer exhibited better stability and could be operated for much longer periods of time. 
Utilizing nanostructures with broad light absorption properties and controllable energy levels to build photovoltaic devices has provided researchers with new strategies to potentially develop next-generation solar cells.[30, 31, 33, 35, 144] The easy tuning of light absorption, sensitivity to diffused light and the ease of processing, starting from solution conditions, have made QDs an important candidate as a light absorber and ideally suited for designing flexible solar panels.[169] Unlike QD-LEDs, the transport of charge carriers in solar cells is kinetically driven not field-driven.[33, 170] Thus, controlling the photoinduced charge separation and their transport across the interface dictates the performance of QD solar cells. By rapidly transferring electrons from the QDs to an acceptor (such as $\mathrm{TiO}_{2}$ or $\mathrm{ZnO}$ ), or by scavenging holes with a redox couple, it is possible to extend the lifetime of the carriers, thus increasing the measured photocurrent. Additional improvements can be realized by minimizing the recombination losses at grain boundaries, which allows better transport of carriers to the collecting electrode, enhancing the power conversion efficiency for the cells.

One popular prototype of QD solar cells currently explored by various groups uses solidstate heterojunction structure, instead of dye sensitized liquid cells (often used with $\mathrm{TiO}_{2}$ materials), as means of charge extraction and for enhancing the electron and hole separation.[35] The majority of those works have centered on the use of PbS and PbSe QDs deposited on $\mathrm{TiO}_{2}$ or $\mathrm{ZnO}$ film that serves as a photoanode. There have been several recent publications reviewing the progress made in developing QD-based solar cells.[31, 33, 35, 171173] We limit our brief discussion to a recent works by the groups of Bawendi and co-workers and Sargent and co-workers.[165, 174] Bawendi and co-workers reported the development of room-temperature solution-processed air-stable $\mathrm{ZnO} / \mathrm{PbS}$ QD solar cells.[165] They used two sets of QDs functionalized with distinct ligands (ligands with different electronic properties) as a means of tuning the relative band alignment in the absorbing layer. The device architecture contained one layer of QD capped with an inorganic ligand (tetrabutylammonium iodide, TBAI), serving as the main light absorbing layer, and a layer of 1,2-ethanedithiol (organic ligand)passivated QDs, serving as an electron-blocking/hole-extraction layer (see Figure 11D). By manipulating the band alignment of the QD layer through the use of different-ligand treatments, an efficiency of $\sim 8.5 \%$ was achieved. Overall, the use of TBAI provided n-type 
quantum dots with remarkable stability against oxidation. The improved stability further allowed the deposition in air of a complementary, p-type PbS quantum dots on the top of TBAI$\mathrm{PbS}$ nanocrystal-layer. The resultant device structure was inverted (compared with previous ones in which the first QD layer of quantum dots constituted p-type PbS), thus producing highperformance quantum dot solar cells with reliable stability in ambient conditions (Figure 11D). In an independent study by Sargent and co-workers, they identified inorganic passivants with strong coordination onto the QD surface, which also repel oxidative attacks.[174] They combined this with an effective processing strategy that can protect the device against protic attack by polar solvents, enabling the assembly of an air-stable n-type solar cell based on PbS QDs. This material design allowed them to build an inverted device, processed in air, with highest current density. Remarkably, they reported quantum efficiency for their solar cell heterostructure ( 8\%) comparable to that reported by Bawendi.

The realization of device architectures with enhanced performance highlights the great impact of using surface chemistry to manipulate and control the electronic properties and stability of various QD materials. This provides an effective tool to ultimately and predictably control the electronic properties of colloidal QD films, and to optimize the performance of QDbased devices. 


\section{Summary and Perspective}

We have summarized some of the various routes used to externally control and modulate the emission of semiconductor QDs via through space interactions that rely on either resonance energy transfer or charge transfer processes. Luminescent QDs offer features that are ideally suited for probing various biological processes, using either mode of interactions. We have also discussed the importance of band alignment on the carrier injection, transport and exciton recombination in light emitting devices, as well as the charge separation and migration in photovoltaic devices. In all the examples discussed the QDs offer the capacity to tune and control those properties, via size and/or composition and manipulation of the surface chemistry.

There have been significant advances in understanding how energy and charge transfer interactions affect the QD photophysical properties, and in exploiting the tunable properties of QDs to optimize those interactions. The use of such interactions as a means to control the QD PL emission has been used in several sensing configurations, most notably sensors with applications that are of interest in biology. The progress made for integrating various QD materials in light emitting and photovoltaic devices has been remarkable, as both device efficiency and color tunability (for LEDs) have continued to improve. These various applications will continue to benefit from better nanomaterials through chemical design, growth conditions, surface ligand chemistry and physics.

One promising idea is to apply some of the QD-conjugates described in the literature for in vivo sensing to detect, for example, changes in $\mathrm{pH}$, ion concentration and ion flux, biomolecular interactions, and membrane-specific processes. Indeed, monitoring $\mathrm{pH}$, changes in intracellular ion concentration and ion fluxes are greatly important for studying a variety of biological processes and diseases.[175] Large changes in $\mathrm{pH}$, or abnormal $\mathrm{pH}$ values or/and patterns are often associated with incorrect cell function and division, which is the case in cancerous tissues. Some of these nanoparticle-conjugates also provide novel intravascular probes for both diagnostic (e.g., imaging) and therapeutic purposes (e.g., drug delivery).

Even as QDs begin to be introduced in mainstream markets, there remain key challenges facing QDs in LEDs and solar cells. For instance, the balance between FRET and direct charge 
injection in the electrical excitation of QD-LEDs remains unclear.[34] More importantly, developing nanoparticles as sensors and delivery platforms is extremely promising, but issues of potential toxicity remain paramount. There is a lack of adequate understanding of these issues, and exploration have been rather scattered and often ineffective as materials with sub optimal stability and controlled properties have been used in several studies. One of those challenges is to understand the operating mechanisms since higher efficiency are predictable from theory. These indicate that the field of nanoparticle research is still very promising both for fundamental exploration as well as for developing new applications.

\section{Acknowledgements}

The authors thank Florida State University, the National Science Foundation for financial support (NSF-CHE \#1058957) and Pfizer for financial support. We also thank Naiqian Zhan and Goutam Palui for the fruitful discussions 


\section{References}

[1] C.B. Murray, C.R. Kagan, M.G. Bawendi, Annu Rev Mater Sci, 30 (2000) 545-610.

[2] A.L. Rogach, D.V. Talapin, E.V. Shevchenko, A. Kornowski, M. Haase, H. Weller, Adv Funct Mater, 12 (2002) 653664.

[3] X.Y. Wu, H.J. Liu, J.Q. Liu, K.N. Haley, J.A. Treadway, J.P. Larson, et al, Nat Biotechnol, 21 (2003) 452-452.

[4] M.C. Daniel, D. Astruc, Chem Rev, 104 (2004) 293-346.

[5] I.L. Medintz, H.T. Uyeda, E.R. Goldman, H. Mattoussi, Nat Mater, 4 (2005) 435-446.

[6] X. Michalet, F.F. Pinaud, L.A. Bentolila, J.M. Tsay, S. Doose, J.J. Li, et al, Science, 307 (2005) 538-544.

[7] R.C. Somers, M.G. Bawendi, D.G. Nocera, Chem Soc Rev, 36 (2007) 579-591.

[8] J. Park, J. Joo, S.G. Kwon, Y. Jang, T. Hyeon, Angew Chem Int Edit, 46 (2007) 4630-4660.

[9] P.K. Jain, X.H. Huang, I.H. El-Sayed, M.A. El-Sayed, Accounts of chemical research, 41 (2008) 1578-1586.

[10] J. Cheon, J.H. Lee, Accounts of chemical research, 41 (2008) 1630-1640.

[11] D.V. Talapin, J.S. Lee, M.V. Kovalenko, E.V. Shevchenko, Chem Rev, 110 (2010) 389-458.

[12] S.S. Agasti, S. Rana, M.H. Park, C.K. Kim, C.C. You, V.M. Rotello, Advanced Drug Delivery Reviews, 62 (2010) 316-328.

[13] V.I. Klimov, Nanocrystal quantum dots, 2nd ed., CRC Press, Boca Raton, 2010.

[14] P. Zrazhevskiy, M. Sena, X.H. Gao, Chem Soc Rev, 39 (2010) 4326-4354.

[15] N. Lee, T. Hyeon, Chem Soc Rev, 41 (2012) 2575-2589.

[16] R. Freeman, I. Willner, Chem Soc Rev, 41 (2012) 4067-4085.

[17] H. Mattoussi, G. Palui, H.B. Na, Adv Drug Deliver Rev, 64 (2012) 138-166.

[18] K.E. Sapsford, W.R. Algar, L. Berti, K.B. Gemmill, B.J. Casey, E. Oh, et al, Chem Rev, 113 (2013) 1904-2074.

[19] P.D. Howes, R. Chandrawati, M.M. Stevens, Science, 346 (2014) 1247390.

[20] S. Silvi, A. Credi, Chem Soc Rev, 44 (2015) 4275-4289.

[21] C.B. Murray, D.J. Norris, M.G. Bawendi, J Am Chem Soc, 115 (1993) 8706-8715.

[22] M.A. Hines, P. Guyot-Sionnest, J Phys Chem-Us, 100 (1996) 468-471.

[23] B.O. Dabbousi, J. RodriguezViejo, F.V. Mikulec, J.R. Heine, H. Mattoussi, R. Ober, et al, J Phys Chem B, 101 (1997) 9463-9475.

[24] X.G. Peng, L. Manna, W.D. Yang, J. Wickham, E. Scher, A. Kadavanich, A.P. Alivisatos, Nature, 404 (2000) 59-61.

[25] Z.A. Peng, X.G. Peng, J Am Chem Soc, 123 (2001) 183-184.

[26] D.V. Talapin, A.L. Rogach, A. Kornowski, M. Haase, H. Weller, Nano Lett, 1 (2001) 207-211.

[27] P. Reiss, J. Bleuse, A. Pron, Nano Lett, 2 (2002) 781-784.

[28] P. Reiss, M. Protière, L. Li, Small, 5 (2009) 154-168.

[29] C.J. Wang, M. Shim, P. Guyot-Sionnest, Science, 291 (2001) 2390-2392.

[30] I. Mora-Sero, S. Gimenez, F. Fabregat-Santiago, R. Gomez, Q. Shen, T. Toyoda, et al, Accounts of chemical research, 42 (2009) 1848-1857. 
[31] F. Hetsch, X.Q. Xu, H.K. Wang, S.V. Kershaw, A.L. Rogach, J Phys Chem Lett, 2 (2011) 1879-1887.

[32] Y. Shirasaki, G.J. Supran, M.G. Bawendi, V. Bulovic, Nat Photonics, 7 (2013) 13-23.

[33] P.V. Kamat, J Phys Chem Lett, 4 (2013) 908-918.

[34] G.J. Supran, Y. Shirasaki, K.W. Song, J.M. Caruge, P.T. Kazlas, S. Coe-Sullivan, et al, Mrs Bull, 38 (2013) $703-711$.

[35] X. Lan, S. Masala, E.H. Sargent, Nat Mater, 13 (2014) 233-240.

[36] C.M. Lemon, P.N. Curtin, R.C. Somers, A.B. Greytak, R.M. Lanning, R.K. Jain, et al, Inorg Chem, 53 (2014) 19001915.

[37] D.M. Adams, L. Brus, C.E.D. Chidsey, S. Creager, C. Creutz, C.R. Kagan, et al, J Phys Chem B, 107 (2003) 66686697.

[38] I. Yildiz, E. Deniz, F.M. Raymo, Chem Soc Rev, 38 (2009) 1859-1867.

[39] I.L. Medintz, H. Mattoussi, Physical Chemistry Chemical Physics, 11 (2009) 17-45.

[40] H.M. Zhu, Y. Yang, T.Q. Lian, Accounts of chemical research, 46 (2013) 1270-1279.

[41] D.A. Hines, P.V. Kamat, Acs Appl Mater Inter, 6 (2014) 3041-3057.

[42] J.K. Jaiswal, H. Mattoussi, J.M. Mauro, S.M. Simon, Nat Biotechnol, 21 (2003) 47-51.

[43] B. Hajj, M. El Beheiry, I. Izeddin, X. Darzacq, M. Dahan, Physical Chemistry Chemical Physics, 16 (2014) 1634016348.

[44] C.M. Tyrakowski, P.T. Snee, Physical Chemistry Chemical Physics, 16 (2014) 837-855.

[45] C.E. Probst, P. Zrazhevskiy, V. Bagalkot, X.H. Gao, Adv Drug Deliver Rev, 65 (2013) 703-718.

[46] E. Cassette, M. Helle, L. Bezdetnaya, F. Marchal, B. Dubertret, T. Pons, Advanced Drug Delivery Reviews, 65 (2013) 719-731.

[47] J. Nam, N. Won, J. Bang, H. Jin, J. Park, S. Jung, et al, Advanced Drug Delivery Reviews, 65 (2013) 622-648.

[48] I.L. Medintz, T. Pons, S.A. Trammell, A.F. Grimes, D.S. English, J.B. Blanco-Canosa, et al, J Am Chem Soc, 130 (2008) 16745-16756.

[49] X. Ji, G. Palui, T. Avellini, H.B. Na, C.Y. Yi, K.L. Knappenberger, et al, J Am Chem Soc, 134 (2012) 6006-6017.

[50] I.L. Medintz, M.H. Stewart, S.A. Trammell, K. Susumu, J.B. Delehanty, B.C. Mei, et al, Nat Mater, 9 (2010) 676684.

[51] X. Ji, N.S. Makarov, W. Wang, G. Palui, I. Robel, H. Mattoussi, The Journal of Physical Chemistry C, 119 (2015) 3388-3399.

[52] X. Ji, W. Wang, H. Mattoussi, Physical chemistry chemical physics : PCCP, 17 (2015) 10108-10117.

[53] C. Burda, S. Link, M. Mohamed, M. El-Sayed, J Phys Chem B, 105 (2001) 12286-12292.

[54] P. Kambhampati, Accounts of chemical research, 44 (2011) 1-13.

[55] L.A. Padilha, J.T. Stewart, R.L. Sandberg, W.K. Bae, W.K. Koh, J.M. Pietryga, et al, Accounts of chemical research, 46 (2013) 1261-1269.

[56] J.R. Lakowicz, Principles of fluorescence spectroscopy, 3rd ed., Springer, New York, 2006.

[57] R.A. Marcus, The Journal of chemical physics, 24 (1956) 966-978.

[58] F.D. Lewis, R.L. Letsinger, M.R. Wasielewski, Accounts of chemical research, 34 (2001) 159-170.

[59] K. Kawai, T. Majima, Accounts of chemical research, 46 (2013) 2616-2625.

[60] N. Zhang, S.R. Samanta, B.M. Rosen, V. Percec, Chem Rev, 114 (2014) 5848-5958. 
[61] C. Narth, N. Gillet, F. Cailliez, B. Lévy, A. de la Lande, Accounts of chemical research, 48 (2015) 1090-1097.

[62] T. Sakata, K. Hashimoto, M. Hiramoto, The Journal of Physical Chemistry, 94 (1990) 3040-3045.

[63] K. Tvrdy, P.A. Frantsuzov, P.V. Kamat, Proceedings of the National Academy of Sciences, 108 (2011) 29-34.

[64] J. Huang, D. Stockwell, Z.Q. Huang, D.L. Mohler, T.Q. Lian, J Am Chem Soc, 130 (2008) 5632-5633.

[65] F.D. Lewis, T. Wu, Y. Zhang, R.L. Letsinger, S.R. Greenfield, M.R. Wasielewski, Science, 277 (1997) 673-676.

[66] W.B. Davis, W.A. Svec, M.A. Ratner, M.R. Wasielewski, Nature, 396 (1998) 60-63.

[67] H.L. Tavernier, M.D. Fayer, The Journal of Physical Chemistry B, 104 (2000) 11541-11550.

[68] J.J. Ratner M. A., Molecular Electronics, (1997) pp 5-72.

[69] I. Robel, M. Kuno, P.V. Kamat, J Am Chem Soc, 129 (2007) 4136-4137.

[70] H. Zhu, N. Song, T. Lian, J Am Chem Soc, 135 (2013) 11461-11464.

[71] U. Resch-Genger, M. Grabolle, S. Cavaliere-Jaricot, R. Nitschke, T. Nann, Nature methods, 5 (2008) 763-775.

[72] E.R. Kay, J. Lee, D.G. Nocera, M.G. Bawendi, Angew Chem Int Edit, 52 (2013) 1165-1169.

[73] A.L. Efros, M. Rosen, Annu Rev Mater Sci, 30 (2000) 475-521.

[74] A.D. Yoffe, Adv Phys, 50 (2001) 1-208.

[75] S.V. Gaponenko, Optical properties of semiconductor nanocrystals, Digitally printed 1st pbk. ed., Cambridge University Press, Cambridge ; New York, 2005.

[76] A.R. Clapp, I.L. Medintz, J.M. Mauro, B.R. Fisher, M.G. Bawendi, H. Mattoussi, J Am Chem Soc, 126 (2004) 301310.

[77] A.M. Dennis, G. Bao, Nano Lett, 8 (2008) 1439-1445.

[78] I.L. Medintz, A.R. Clapp, H. Mattoussi, E.R. Goldman, B. Fisher, J.M. Mauro, Nat Mater, 2 (2003) 630-638.

[79] A.R. Clapp, I.L. Medintz, H.T. Uyeda, B.R. Fisher, E.R. Goldman, M.G. Bawendi, et al, J Am Chem Soc, 127 (2005) 18212-18221.

[80] H. Lu, O. Schöps, U. Woggon, C.M. Niemeyer, J Am Chem Soc, 130 (2008) 4815-4827.

[81] P.T. Snee, C.M. Tyrakowski, L.E. Page, A. Isovic, A.M. Jawaid, The Journal of Physical Chemistry C, 115 (2011) 19578-19582.

[82] N.Q. Zhan, G. Palui, M. Safi, X. Ji, H. Mattoussi, J Am Chem Soc, 135 (2013) 13786-13795.

[83] T. Pons, I.L. Medintz, X. Wang, D.S. English, H. Mattoussi, J Am Chem Soc, 128 (2006) 15324-15331.

[84] A. Kurkdjian, J. Guern, Annu Rev Plant Phys, 40 (1989) 271-303.

[85] P.D. Boyer, Annu Rev Biochem, 66 (1997) 717-749.

[86] A.M. Dennis, W.J. Rhee, D. Sotto, S.N. Dublin, G. Bao, Acs Nano, 6 (2012) 2917-2924.

[87] R. Tang, H. Lee, S. Achilefu, J Am Chem Soc, 134 (2012) 4545-4548.

[88] P.T. Snee, R.C. Somers, G. Nair, J.P. Zimmer, M.G. Bawendi, D.G. Nocera, J Am Chem Soc, 128 (2006) 1332013321.

[89] W. Wang, A. Kapur, X. Ji, M. Safi, G. Palui, V. Palomo, et al, J Am Chem Soc, 137 (2015) 5438-5451.

[90] A.I. Zamaleeva, M. Collot, E. Bahembera, C. Tisseyre, P. Rostaing, A.V. Yakovlev, et al, Nano Lett, 14 (2014) 2994-3001.

[91] W.A. Catterall, Neuron, 26 (2000) 13-25. 
[92] B. Hille, Ion channels of excitable membranes, Sinauer Sunderland, MA, 2001.

[93] E. Gouaux, R. MacKinnon, Science, 310 (2005) 1461-1465.

[94] R. Freeman, T. Finder, I. Willner, Angew Chem Int Edit, 48 (2009) 7818-7821.

[95] E.R. Goldman, I.L. Medintz, J.L. Whitley, A. Hayhurst, A.R. Clapp, H.T. Uyeda, et al, J Am Chem Soc, 127 (2005) 6744-6751.

[96] V.W. Yong, C. Power, P. Forsyth, D.R. Edwards, Nature reviews. Neuroscience, 2 (2001) 502-511.

[97] B. Turk, Nat Rev Drug Discov, 5 (2006) 785-799.

[98] S.P. Jackson, J. Bartek, Nature, 461 (2009) 1071-1078.

[99] M.A. Rizzo, G.H. Springer, B. Granada, D.W. Piston, Nat Biotech, 22 (2004) 445-449.

[100] T. Kohl, K.G. Heinze, R. Kuhlemann, A. Koltermann, P. Schwille, Proceedings of the National Academy of Sciences, 99 (2002) 12161-12166.

[101] R. Gill, R. Freeman, J.P. Xu, I. Willner, S. Winograd, I. Shweky, et al, J Am Chem Soc, 128 (2006) 15376-15377.

[102] M. Suzuki, Y. Husimi, H. Komatsu, K. Suzuki, K.T. Douglas, J Am Chem Soc, 130 (2008) 5720-5725.

[103] Z.Y. Xia, Y. Xing, M.K. So, A.L. Koh, R. Sinclair, J.H. Rao, Analytical chemistry, 80 (2008) 8649-8655.

[104] K. Boeneman, B.C. Mei, A.M. Dennis, G. Bao, J.R. Deschamps, H. Mattoussi, et al, J Am Chem Soc, 131 (2009) 3828-3829.

[105] S.B. Lowe, J.A.G. Dick, B.E. Cohen, M.M. Stevens, ACS nano, 6 (2012) 851-857.

[106] W.R. Algar, A.J. Tavares, U.J. Krull, Analytica chimica acta, 673 (2010) 1-25.

[107] K.E. Sapsford, D. Farrell, S. Sun, A. Rasooly, H. Mattoussi, I.L. Medintz, Sensor Actuat B-Chem, 139 (2009) 1321.

[108] J.E. Ghadiali, B.E. Cohen, M.M. Stevens, ACS nano, 4 (2010) 4915-4919.

[109] W.R. Algar, A. Malonoski, J.R. Deschamps, J.B. Blanco-Canosa, K. Susumu, M.H. Stewart, et al, Nano Lett, 12 (2012) 3793-3802.

[110] L.F. Shi, V. De Paoli, N. Rosenzweig, Z. Rosenzweig, J Am Chem Soc, 128 (2006) 10378-10379.

[111] I.L. Medintz, A.R. Clapp, F.M. Brunel, T. Tiefenbrunn, H.T. Uyeda, E.L. Chang, et al, Nat Mater, 5 (2006) 581589.

[112] E.Y. Chung, C.J. Ochs, Y. Wang, L. Lei, Q. Qin, A.M. Smith, et al, Nano Lett, 15 (2015) 5025-5032.

[113] C.Y. Zhang, H.C. Yeh, M.T. Kuroki, T.H. Wang, Nat Mater, 4 (2005) 826-831.

[114] E. Sharon, R. Freeman, I. Willner, Analytical chemistry, 82 (2010) 7073-7077.

[115] A. Banerjee, C. Grazon, B. Nadal, T. Pons, Y. Krishnan, B. Dubertret, Bioconjugate chemistry, 26 (2015) 15821589.

[116] M.G. Sandros, D. Gao, D.E. Benson, J Am Chem Soc, 127 (2005) 12198-12199.

[117] B.P. Aryal, D.E. Benson, J Am Chem Soc, 128 (2006) 15986-15987.

[118] T. Nann, S.K. Ibrahim, P.M. Woi, S. Xu, J. Ziegler, C.J. Pickett, Angew Chem Int Ed Engl, 49 (2010) 1574-1577.

[119] Z.J. Han, F. Qiu, R. Eisenberg, P.L. Holland, T.D. Krauss, Science, 338 (2012) 1321-1324.

[120] Z.J. Han, R. Eisenberg, Accounts of chemical research, 47 (2014) 2537-2544.

[121] A.C. Samia, X. Chen, C. Burda, J Am Chem Soc, 125 (2003) 15736-15737.

[122] J.M. Tsay, M. Trzoss, L. Shi, X. Kong, M. Selke, M.E. Jung, et al, J Am Chem Soc, 129 (2007) 6865-6871. 
[123] D. Neuman, A.D. Ostrowski, R.O. Absalonson, G.F. Strouse, P.C. Ford, J Am Chem Soc, 129 (2007) $4146-4147$.

[124] P. Juzenas, W. Chen, Y.P. Sun, M.A. Coelho, R. Generalov, N. Generalova, et al, Adv Drug Deliver Rev, 60 (2008) 1600-1614.

[125] V. Biju, S. Mundayoor, R.V. Omkumar, A. Anas, M. Ishikawa, Biotechnology advances, 28 (2010) $199-213$.

[126] P.C. Ford, Accounts of chemical research, 41 (2008) 190-200.

[127] P.T. Burks, A.D. Ostrowski, A.A. Mikhailovsky, E.M. Chan, P.S. Wagenknecht, P.C. Ford, J Am Chem Soc, 134 (2012) 13266-13275.

[128] M.P. Singh, G.F. Strouse, J Am Chem Soc, 132 (2010) 9383-9391.

[129] C.J. Breshike, R.A. Riskowski, G.F. Strouse, The Journal of Physical Chemistry C, 117 (2013) 23942-23949.

[130] E. Oh, M.Y. Hong, D. Lee, S.H. Nam, H.C. Yoon, H.S. Kim, J Am Chem Soc, 127 (2005) 3270-3271.

[131] J.W. Liu, J.H. Lee, Y. Lu, Analytical chemistry, 79 (2007) 4120-4125.

[132] X. Zhang, C.A. Marocico, M. Lunz, V.A. Gerard, Y.K. Gun'ko, V. Lesnyak, et al, ACS nano, 6 (2012) 9283-9290.

[133] H. Han, V. Valle, M.M. Maye, J Phys Chem C, 116 (2012) 22996-23003.

[134] M. Li, S.K. Cushing, Q.Y. Wang, X.D. Shi, L.A. Hornak, Z.L. Hong, et al, J Phys Chem Lett, 2 (2011) 2125-2129.

[135] T. Pons, I.L. Medintz, K.E. Sapsford, S. Higashiya, A.F. Grimes, D.S. English, et al, Nano Lett, 7 (2007) 31573164.

[136] D. Nepal, L.F. Drummy, S. Biswas, K. Park, R.A. Vaia, ACS nano, 7 (2013) 9064-9074.

[137] N.S. Abadeer, M.R. Brennan, W.L. Wilson, C.J. Murphy, ACS nano, 8 (2014) 8392-8406.

[138] O. Kulakovich, N. Strekal, A. Yaroshevich, S. Maskevich, S. Gaponenko, I. Nabiev, et al, Nano Lett, 2 (2002) 1449-1452.

[139] K. Munechika, Y. Chen, A.F. Tillack, A.P. Kulkarni, I.J.-L. Plante, A.M. Munro, et al, Nano Lett, 10 (2010) $2598-$ 2603.

[140] S.-H. Gong, J.-H. Kim, Y.-H. Ko, C. Rodriguez, J. Shin, Y.-H. Lee, et al, Proceedings of the National Academy of Sciences, 112 (2015) 5280-5285.

[141] V.K. Komarala, A.L. Bradley, Y.P. Rakovich, S.J. Byrne, Y.K. Gun'ko, A.L. Rogach, Appl Phys Lett, 93 (2008) 123102.

[142] M. Lunz, V.A. Gerard, Y.K. Gun'ko, V. Lesnyak, N. Gaponik, A.S. Susha, et al, Nano Lett, 11 (2011) 3341-3345.

[143] C.H. Wang, C.W. Chen, Y.T. Chen, C.M. Wei, Y.F. Chen, C.W. Lai, et al, Appl Phys Lett, 96 (2010) 071906.

[144] P.V. Kamat, J Phys Chem C, 116 (2012) 11849-11851.

[145] J.I. Basham, G.K. Mor, C.A. Grimes, ACS nano, 4 (2010) 1253-1258.

[146] J.M. Haremza, M.A. Hahn, T.D. Krauss, Nano Lett, 2 (2002) 1253-1258.

[147] F. Li, D.I. Son, T.W. Kim, E. Ryu, S.W. Kim, S.K. Lee, Y.H. Cho, Appl Phys Lett, 95 (2009) 061911.

[148] Z.Y. Chen, S. Berciaud, C. Nuckolls, T.F. Heinz, L.E. Brus, ACS nano, 4 (2010) 2964-2968.

[149] C.X. Guo, H.B. Yang, Z.M. Sheng, Z.S. Lu, Q.L. Song, C.M. Li, Angew Chem Int Edit, 49 (2010) 3014-3017.

[150] S. Jander, A. Kornowski, H. Weller, Nano Lett, 11 (2011) 5179-5183.

[151] E. Morales-Narvaez, B. Perez-Lopez, L.B. Pires, A. Merkoci, Carbon, 50 (2012) 2987-2993.

[152] K.I. Bolotin, K.J. Sikes, Z. Jiang, M. Klima, G. Fudenberg, J. Hone, et al, Solid State Commun, 146 (2008) $351-$ 355. 
[153] M. Nirmal, B.O. Dabbousi, M.G. Bawendi, J.J. Macklin, J.K. Trautman, T.D. Harris, et al, Nature, 383 (1996) 802-804.

[154] A.L. Efros, M. Rosen, Physical review letters, 78 (1997) 1110-1113.

[155] A.R. Clapp, I.L. Medintz, B.R. Fisher, G.P. Anderson, H. Mattoussi, J Am Chem Soc, 127 (2005) 1242-1250.

[156] C.R. Kagan, C.B. Murray, M.G. Bawendi, Phys Rev B, 54 (1996) 8633-8643.

[157] S.A. Crooker, J.A. Hollingsworth, S. Tretiak, V.I. Klimov, Physical review letters, 89 (2002) 186802.

[158] N. Hildebrandt, L.J. Charbonniere, M. Beck, R.F. Ziessel, H.G. Lohmannsroben, Angew Chem Int Edit, 44 (2005) 7612-7615.

[159] D. Geissler, L.J. Charbonniere, R.F. Ziessel, N.G. Butlin, H.G. Lohmannsroben, N. Hildebrandt, Angew Chem Int Edit, 49 (2010) 1396-1401.

[160] W.R. Algar, D. Wegner, A.L. Huston, J.B. Blanco-Canosa, M.H. Stewart, A. Armstrong, et al, J Am Chem Soc, 134 (2012) 1876-1891.

[161] X. Qiu, N. Hildebrandt, ACS nano, 9 (2015) 8449-8457.

[162] M.K. So, C.J. Xu, A.M. Loening, S.S. Gambhir, J.H. Rao, Nat Biotechnol, 24 (2006) 339-343.

[163] H.Q. Yao, Y. Zhang, F. Xiao, Z.Y. Xia, J.H. Rao, Angew Chem Int Edit, 46 (2007) 4346-4349.

[164] R. Freeman, X.Q. Liu, I. Willner, J Am Chem Soc, 133 (2011) 11597-11604.

[165] C.H.M. Chuang, P.R. Brown, V. Bulovic, M.G. Bawendi, Nat Mater, 13 (2014) 796-801.

[166] X. Dai, Z. Zhang, Y. Jin, Y. Niu, H. Cao, X. Liang, et al, Nature, 515 (2014) 96-99.

[167] B.S. Mashford, M. Stevenson, Z. Popovic, C. Hamilton, Z.Q. Zhou, C. Breen, J. Steckel, et al, Nat Photonics, 7 (2013) 407-412.

[168] J. Kwak, W.K. Bae, D. Lee, I. Park, J. Lim, M. Park, et al, Nano Lett, 12 (2012) 2362-2366.

[169] P.V. Kamat, K. Tvrdy, D.R. Baker, J.G. Radich, Chem Rev, 110 (2010) 6664-6688.

[170] I. Hod, V. Gonzalez-Pedro, Z. Tachan, F. Fabregat-Santiago, I. Mora-Sero, J. Bisquert, et al, J Phys Chem Lett, 2 (2011) 3032-3035.

[171] I.J. Kramer, E.H. Sargent, Chem Rev, 114 (2014) 863-882.

[172] S. Ten Cate, C.S. Sandeep, Y. Liu, M. Law, S. Kinge, A.J. Houtepen, et al, Accounts of chemical research, 48 (2015) 174-181.

[173] M.R. Kim, D.L. Ma, J Phys Chem Lett, 6 (2015) 85-99.

[174] Z.J. Ning, O. Voznyy, J. Pan, S. Hoogland, V. Adinolfi, J.X. Xu, et al, Nat Mater, 13 (2014) 822-828.

[175] J.Y. Han, K. Burgess, Chem Rev, 110 (2010) 2709-2728. 


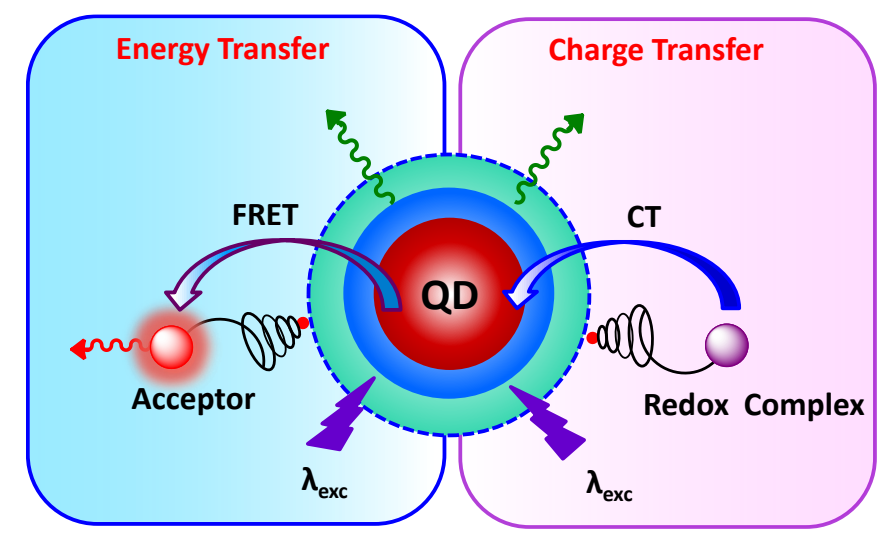

Figure 1. Schematic diagrams showing QD-based energy transfer (left) and charge transfer (right) interactions. 

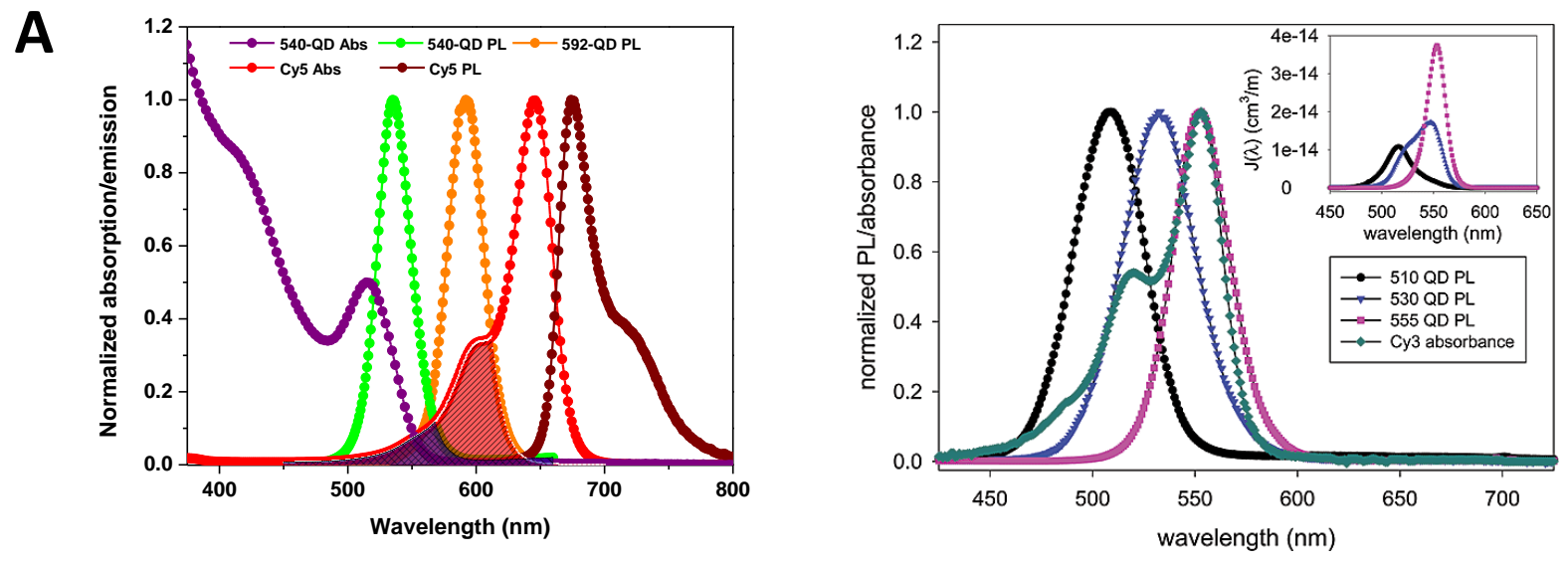

B
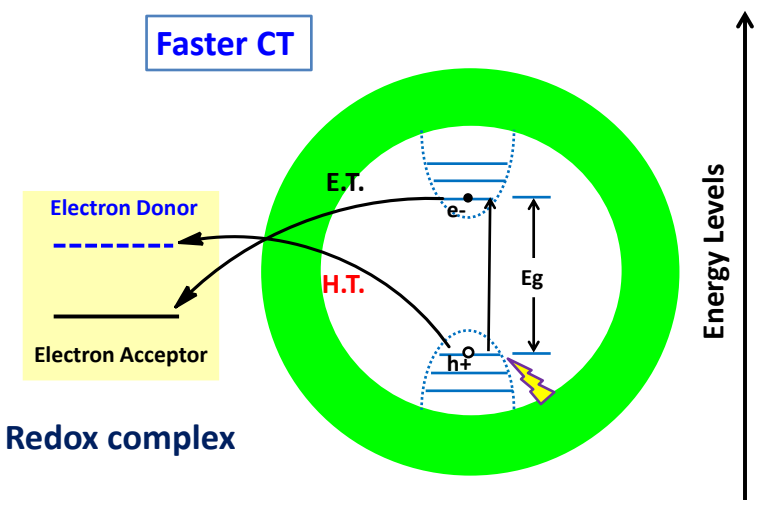

Larger Bandgap

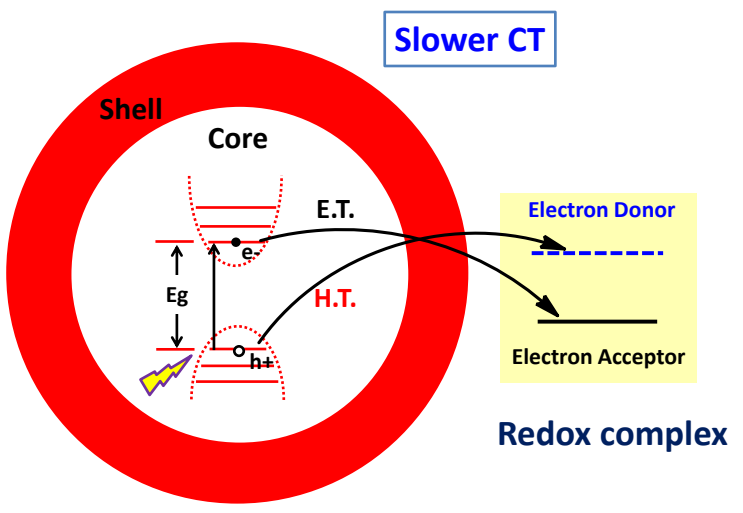

Smaller Bandgap

Figure 2. (A, left) Absorption of 540-nm QDs, emission of 540-nm and 592-nm QDs superimposed over the absorption spectrum of Cy5, along with emission spectrum of Cy5. (A, right) PL spectra for three different-size QDs superposed with the absorption spectrum of Cy3 dye; normalized spectra are shown. Inset shows the corresponding spectral overlap function $J(\lambda)$. The spectra indicate that tuning the QD emission can substantially improve the extent of spectral overlap with the dye. (B) Schematic representation of charge transfer mechanism (involving electron and hole carriers) for the two-size QDs: green-emitting QD with larger bandgap and red-emitting QD with smaller bandgap. Two external pathways are illustrated: (1) hole transfer (H.T.) from the QD to a redox complex (electron donor) and (2) electron transfer (E.T.) from the QD to a redox complex (electron acceptor). Figures partially reproduced from reference,[76] with permissions from the American Chemical Society. 
A
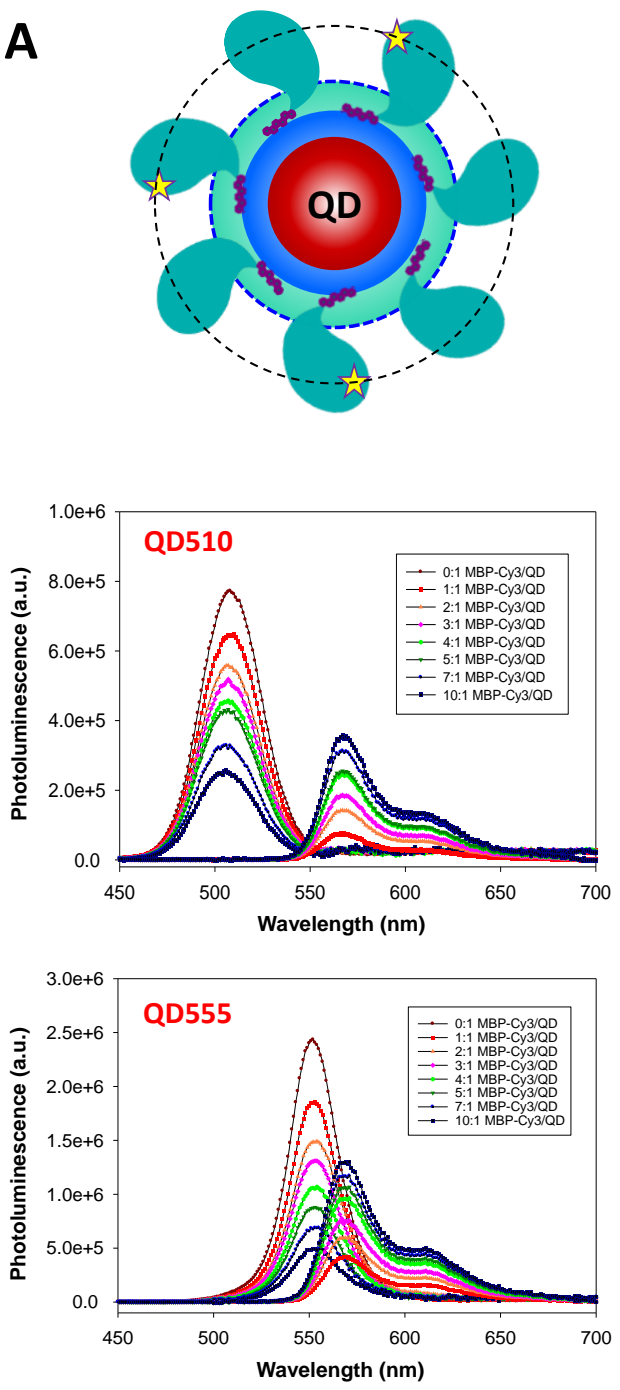

B
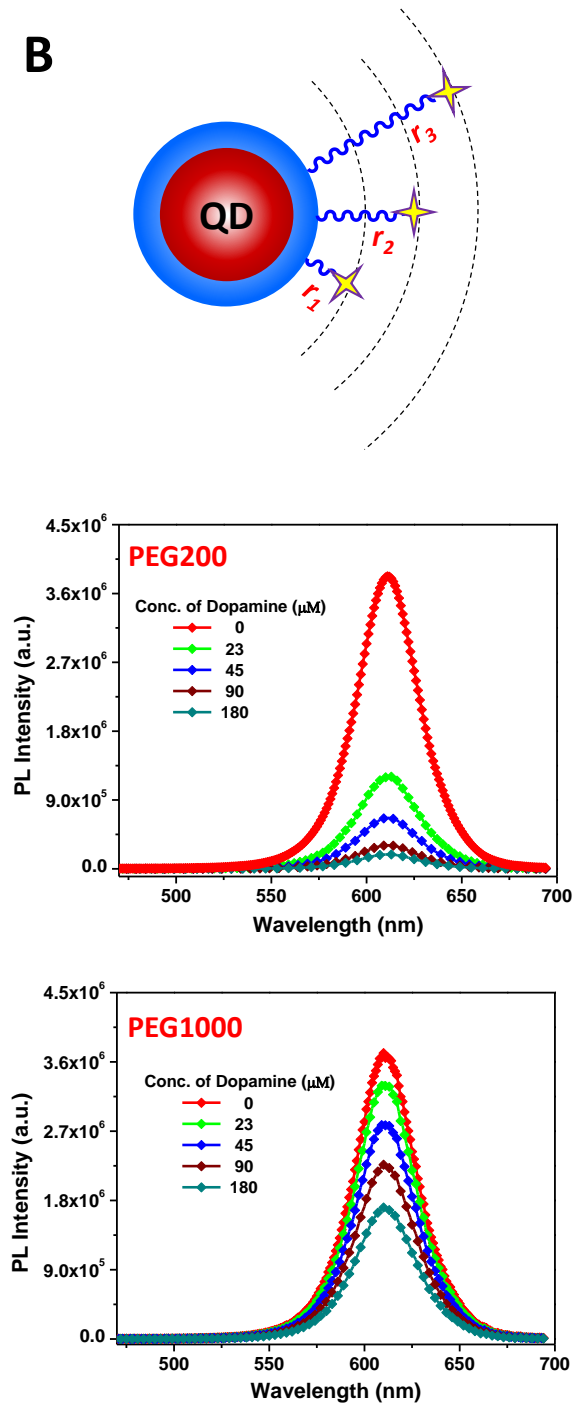

Figure 3. (A) Schematic representation of the QD conjugated to multiple MBP-dye (and MBP) driven by polyhistidine coordination (top). Evolution of the PL spectra of QDs (emitting at 510 and $555 \mathrm{~nm}$ ) and Cy3 (in the assemblies) versus dye-to-QD ratio $n$, after spectral deconvolution, is shown in the middle and bottom panels. The total number of MBP (labeled and unlabeled) per QD conjugate was fixed. A progressive quenching of the QD emission and a systematic enhancement of the Cy3 emission were measured as the number of dyes surrounding the QD increased from 0 to 10. (B) Schematic representation of QD-redox-dopamine assemblies with size-tunable separation distance $\left(r_{1}<r_{2}<r_{3}\right)$. The evolution of the PL spectra of the QDs as function of the added dopamine concentration (i.e., QDconjugate valence) are shown for PEG200 (middle panel) and for PEG1000 (bottom panel). Figures reproduced from references, $[52,76]$ with permissions from the American Chemical Society and the Royal Society of Chemistry. 
A

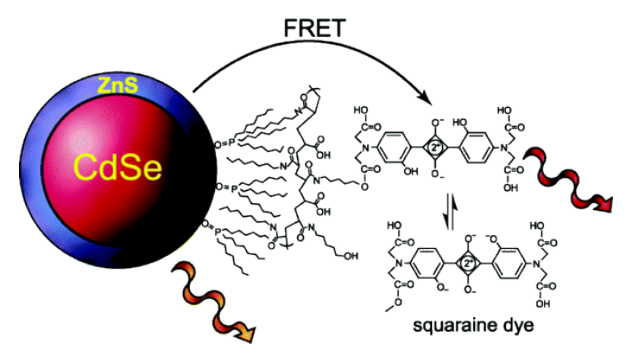

B

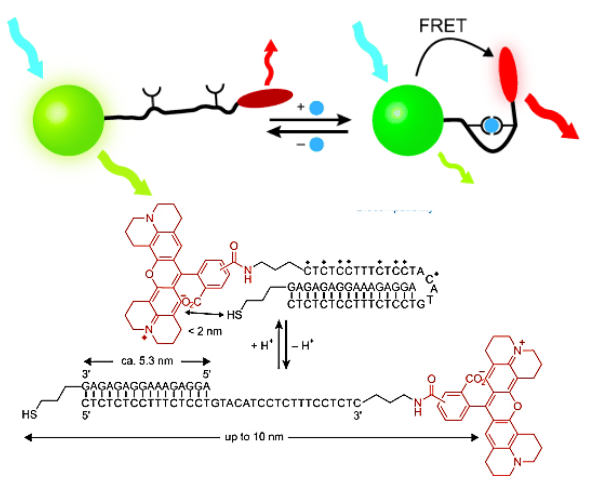

C
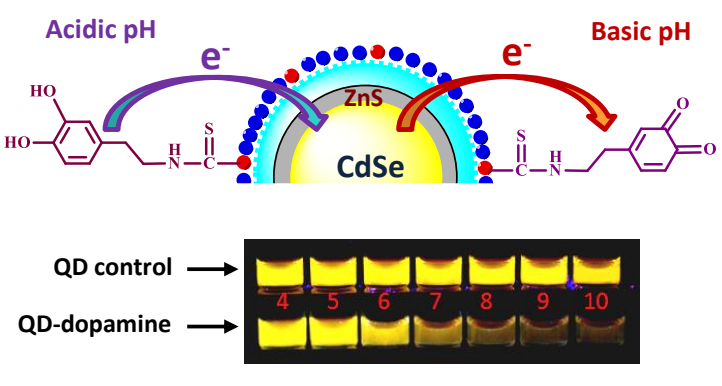
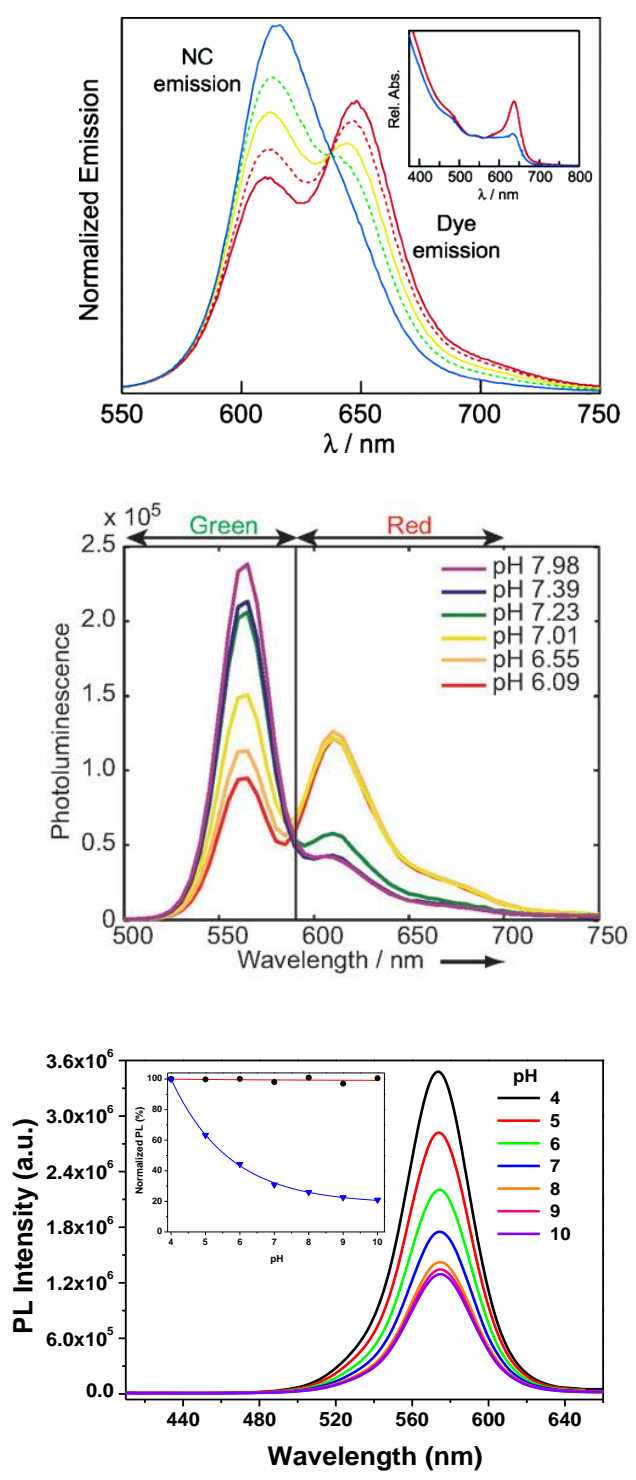

Figure 4. (A) Schematic of a pH sensor constructed using a CdSe-ZnS QD conjugated to a pH-sensitive squaraine dye. FRET interactions are modulated by the environment as the dye absorption varies with $\mathrm{pH}$ from 6 (red) to 10 (blue); absorption of the dye is suppressed at basic $\mathrm{pH}$ as shown in the inset. (B) Schematic illustration of a sensor design wherein a QD is conjugated to carboxy-X-rhodamine through an oligonucleotide linker. Changing the $\mathrm{pH}$ from $\sim 6$ to $\sim 8$ triggers a conformational change in the oligonucleotide structure, altering the donor-acceptor distance and the ensuing FRET efficiency. (C) Schematics representing the $\mathrm{pH}$ modulation of the charge transfer interactions in QD-dopamine conjugates. From acidic to alkaline conditions, there is a $\mathrm{pH}$-induced transformation of dopamine from catechol to quinone. The fluorescence images (in the left panel) show the PL for QDs only (control) and for QD-dopamine conjugates dispersed in different $\mathrm{pH}$ buffers. Emission spectra are collected from dispersions of QD-dopamine conjugates at increasing $\mathrm{pH}$ from 4 to 10. Integrated $\mathrm{PL}$ intensity for the $\mathrm{pH}$ range 4-10 normalized (as percentage) with respect to the initial values at $\mathrm{pH} 4$ is shown in the inset. Figures reproduced from references, $[49,72,88]$ with permissions from the American Chemical Society and Wiley. 

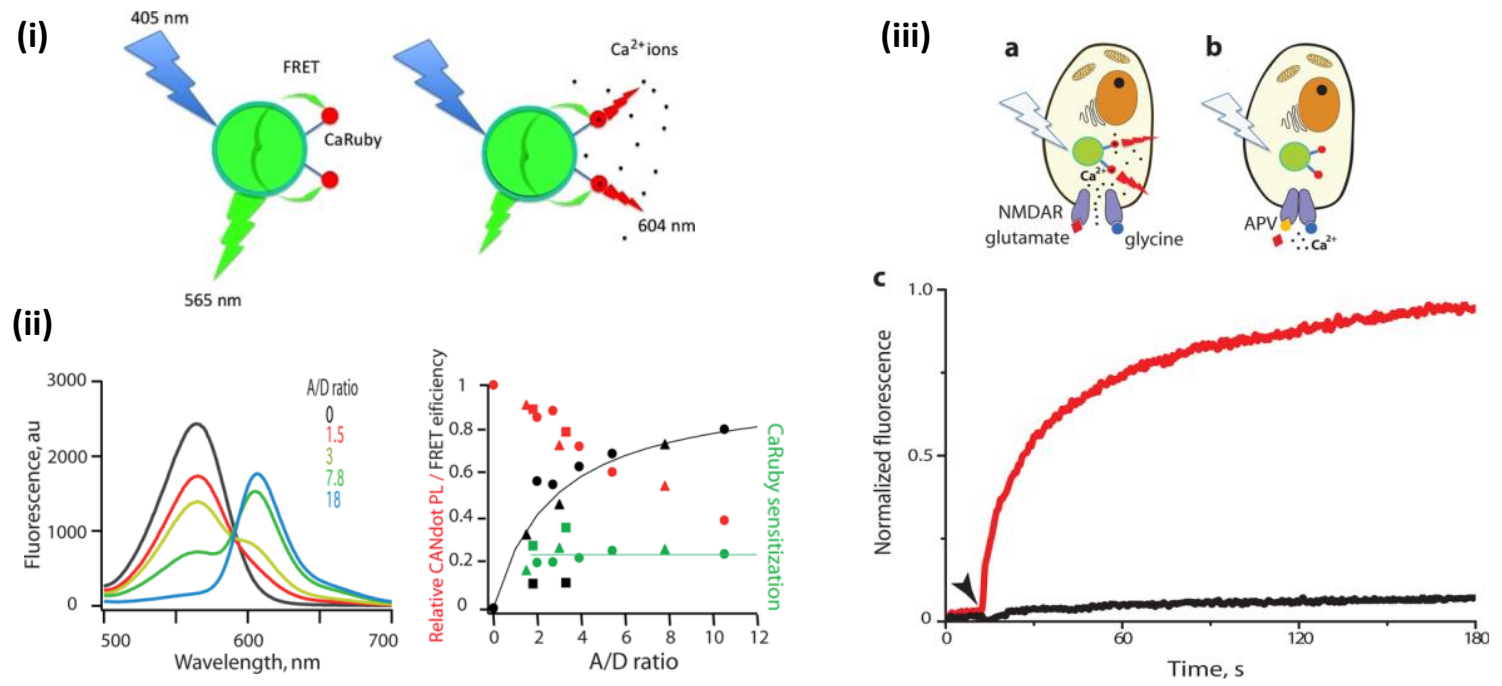

Figure 5. (i) Schematic diagram of the QD-CaRuby conjugates and the $\mathrm{Ca}^{2+}$ sensing mechanism involved. (ii) PL spectra of the QD-CaRuby as a function of CaRuby /QD ratio from 0:1 to 18:1 in the presence of 2 $\mathrm{mM} \mathrm{Ca}{ }^{2+}$ ions. Experimental values for QD PL quenching (red) and FRET efficiency (black), along with acceptor sensitization (green) are reported. (iii) Schematics of the intracellular $\mathrm{Ca}^{2+}$ measurement using a suspension of HEK293 cells transfected with NMDARs and incubated with the conjugates: (a) NMDAR activation by co-application of agonists (glutamate and glycine) followed by $\mathrm{Ca}^{2+}$ influx into the cell; (b) NMDAR inhibition by the antagonist (APV) prevents $\mathrm{Ca}^{2+}$ influx upon application of the agonists; (c) evolution of the PL intensity with time in the presence of intracellular $\mathrm{Ca}^{2+}$ ions. Figures reproduced from references, [90] with permissions from the American Chemical Society. 
A

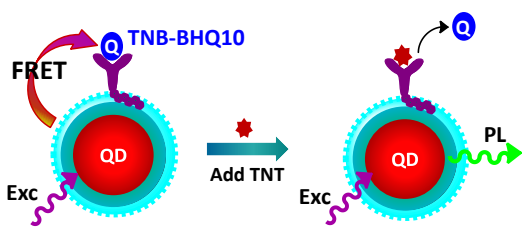

(i)

B

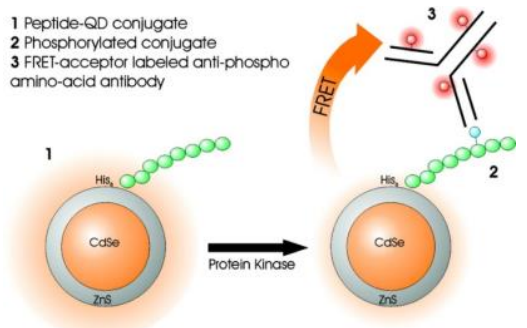

(i)

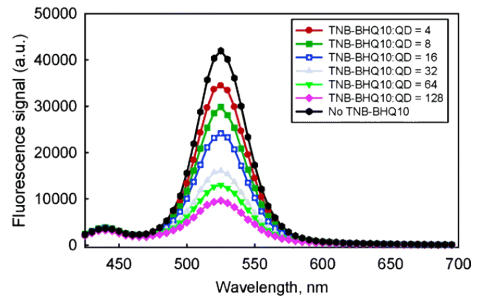

(ii)

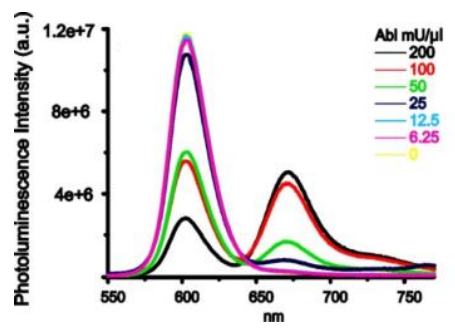

(ii)

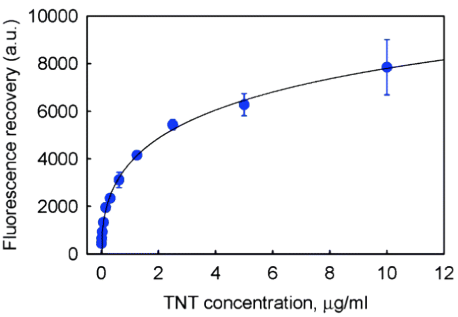

(iii)

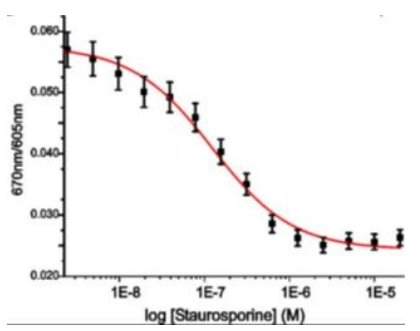

(iii)

C

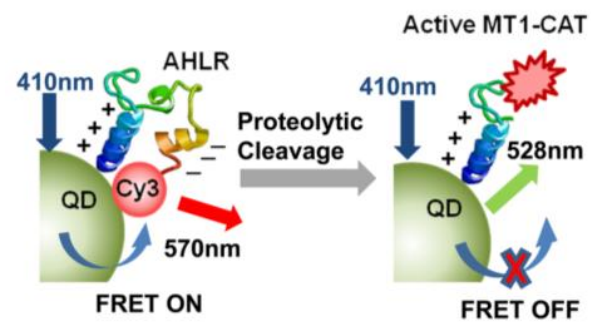

(i)

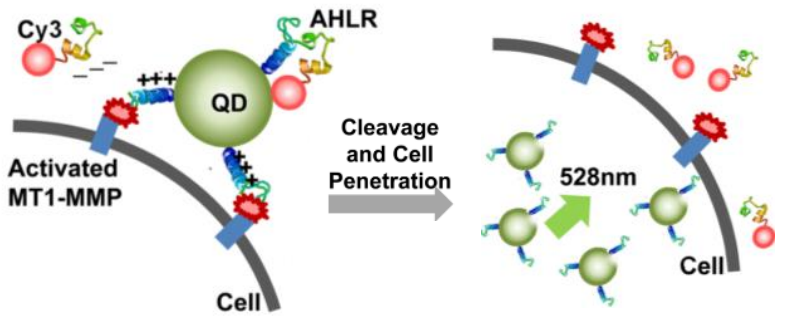

(ii)

Figure 6. (A) Competitive sensing using $Q D$ bioconjugates: (i) schematic representation of the competitive displacement of TNB analog by added TNT using a QD-TNB2-45 assembly; (ii) PL spectra of QD-TNB2-45 conjugates as a function of increasing TNB-BHQ-10 to QD ratio; (iii) the increase in QD PL versus TNT concentration with respect to the control (without TNT). (B) Sensing enzymatic activity using QD-peptide conjugates: (i) schematic representation of kinase-mediated phosphorylation of QD-peptide conjugates, antibody recognition of phosphopeptide, along with FRET-based detection; (ii) steady-state fluorescence spectra ( $\lambda_{\mathrm{ex}}=400 \mathrm{~nm}$ ) of Abl kinase reactions using QD-peptide conjugate substrates after 1 $\mathrm{h}$ of enzyme reaction, followed by the addition of FRET-acceptor labeled antibody; (iii) dose response curve of staurosporine inhibition of Abl activity. (C) QD-FRET nanosensor for profiling MT1-MMP activity in solution and in cell cultures: (i) change in the conjugate structure following enzymatic cleavage of the substrate (AHLR sequence) by MT1-CAT and the corresponding reduction in FRET quenching; (ii) FRET nanosensor activation in the presence of cancer cells expressing MT1-MMP on their membranes. When the AHLR sequence is cleaved, the FRET ratio is increased combined with intracellular uptake of QDs promoted by the arginine peptide sequence. Figures partially reproduced from references, $[95,108,112]$ with permissions from the American Chemical Society. 
A

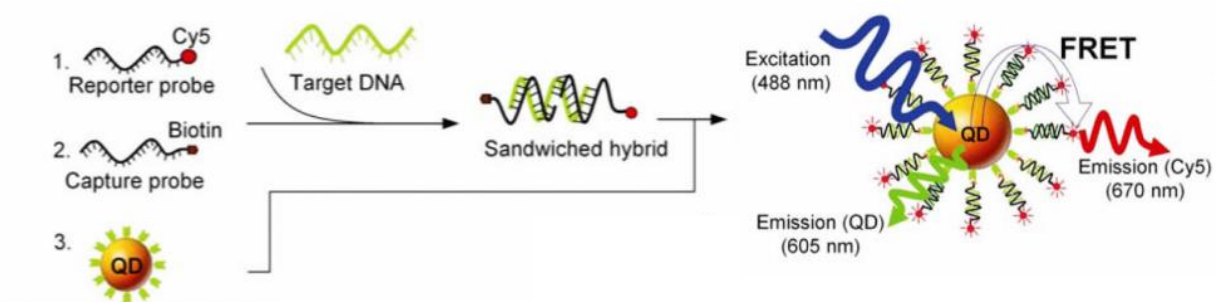

Streptavidin-conjugated QD

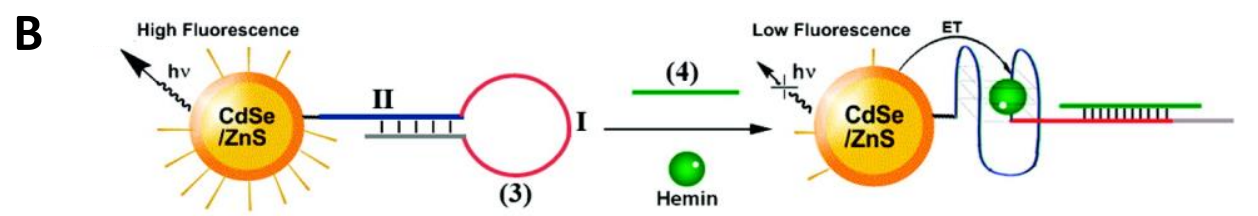

(3) 5'-HS( $\left(\mathrm{CH}_{2}\right)_{6}$-AAGGGTTGGGCGGGATGGGTTACCTCAGTGCTTATTCGAAACCCA-3'

(4) 5'-TCGAATAAGCACTGAGGT-3'

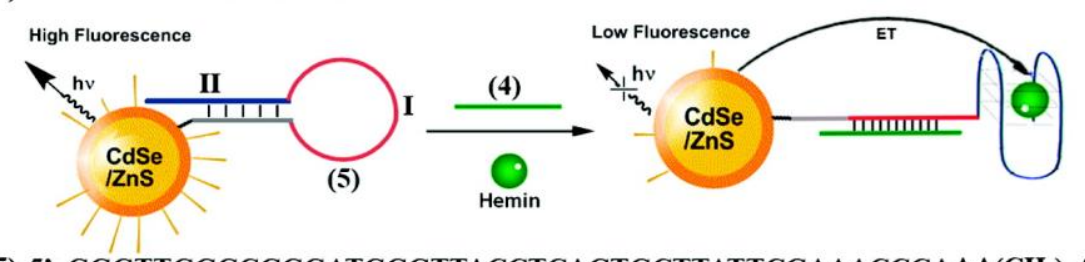

(5) 5'-GGGTTGGGCGGGATGGGTTACCTCAGTGCTTATTCGAAACCCAAA( $\left(\mathrm{CH}_{2}\right)_{6}$-SH-3'

Figure 7. (A) Schematics illustrating the formation of a FRET-based sandwich sensing assembly in the presence of target sequences. Fluorescence from the Cy5 upon excitation of the QD is caused by FRET between the QD donor and Cy5 acceptors in the assembly. (B) Schematic representation of a target nucleic acid via charge transfer interactions using different G-quadruplex-hairpin-modified QDs: Gquadruplex/hemin is formed close to the QDs (top) and G-quadruplex/hemin is formed apart from the QDs (bottom). Figures reproduced from references, $[113,114]$ with permissions from the American Chemical Society and NPG. 
(i)

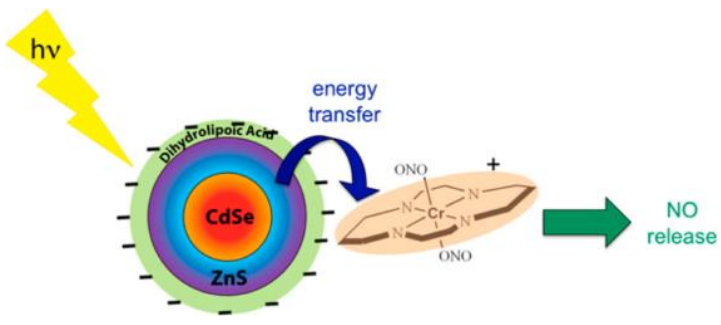

(ii)

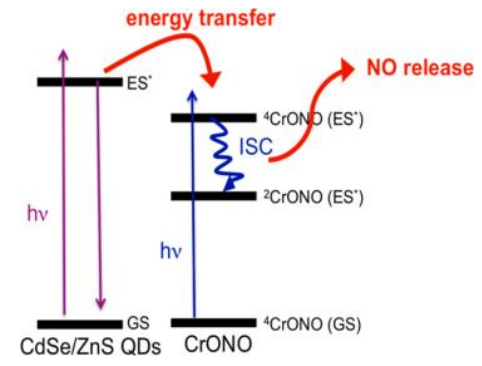

(iii)

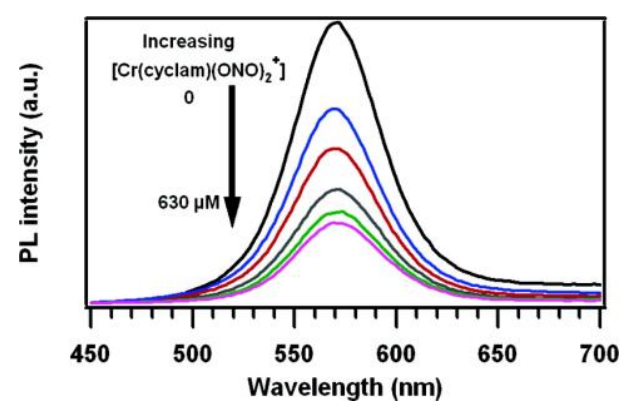

(iv)

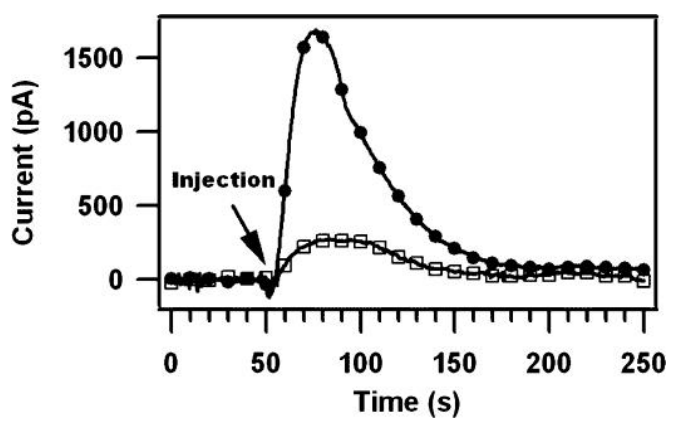

Figure 8. Release of NO promoted by FRET interactions from the QD to $\mathrm{Cr}$ (III) complex, $\mathrm{CrNON}$ : (i) schematic representation of the photosensitized NO release from CrONO, using CrONO adsorbed on the QDs via electrostatic interactions; (ii) mechanism of FRET-induced NO release from the complex; (iii) PL spectra $\left(\lambda_{\text {ex }}=\right.$ $366 \mathrm{~nm}$ ) of water-soluble QDs (130 nM) in phosphate buffer with various concentrations of CrONO (0-630 $\mu \mathrm{M})$, and (iv) comparison of NO production with and without QDs (measured with a NO-specific electrode). Figures partially reproduced from references, $[123,127]$ with permissions from the American Chemical Society. 
A (i)

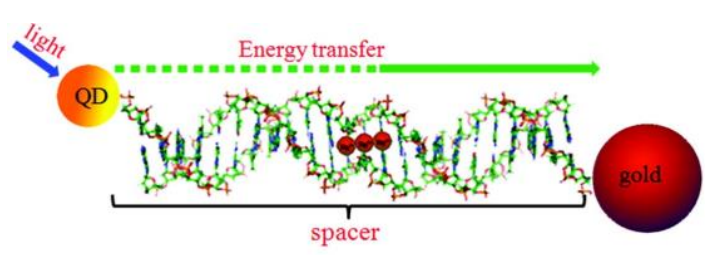

(iii)
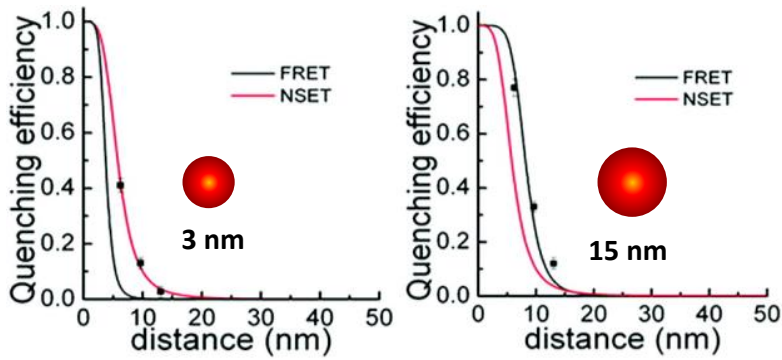

(ii)
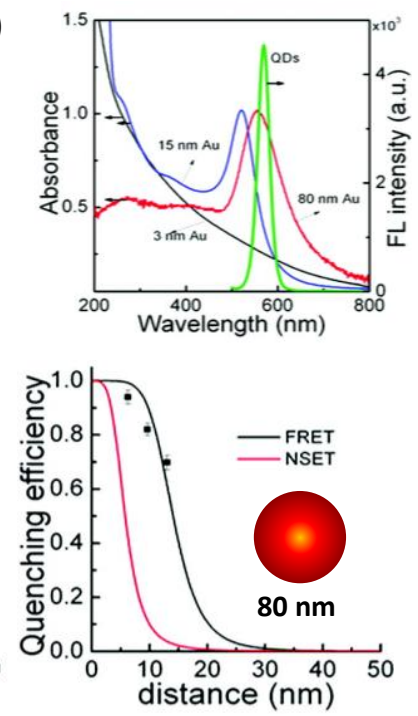

B (i)

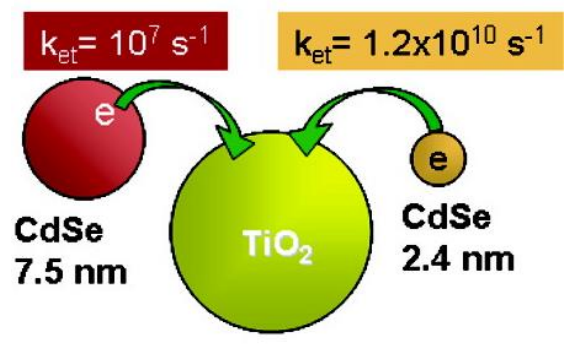

(ii)

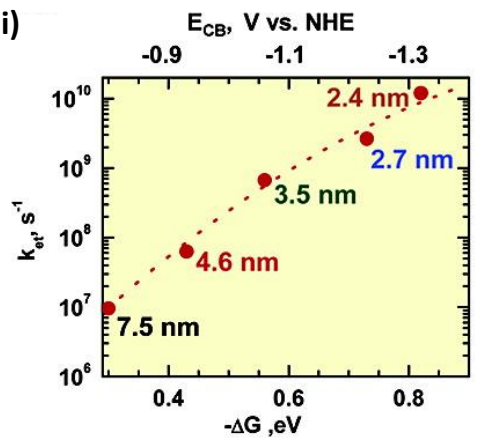

(ii)

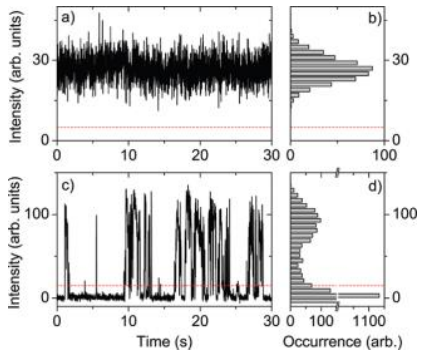

Figure 9. (A) Energy transfer between QDs and different size AuNPs: (i) a rigid double-stranded DNA spacer is used to control the QD-AuNP separation distance; (ii) QD emission spectrum superposed with the absorption spectra of 3, 15 and $80 \mathrm{~nm}$ AuNPs; (iii) quenching efficiency vs. separation distance for the three size AuNPs and the expected quenching for FRET and NSET mechanisms. (B) Charge transfer between $\mathrm{QDs}$ and $\mathrm{TiO}_{2}$ nanocrystal: (i) schematic representation of electron transfer from different size $\mathrm{QD}$ to $\mathrm{TiO}_{2}$; (ii) the dependence of electron transfer rate constant on the QD size. (C) Energy transfer between QDs and graphene probed at the single molecule level: (i) fluorescence image and schematic diagram of QDs on a single-layer graphene and on a quartz substrate; (ii) suppression of the QD PL blinking when immobilized on a single layer of graphene. Figures reprinted from references,[69, 134, 148] with permission of the American Chemical Society. 

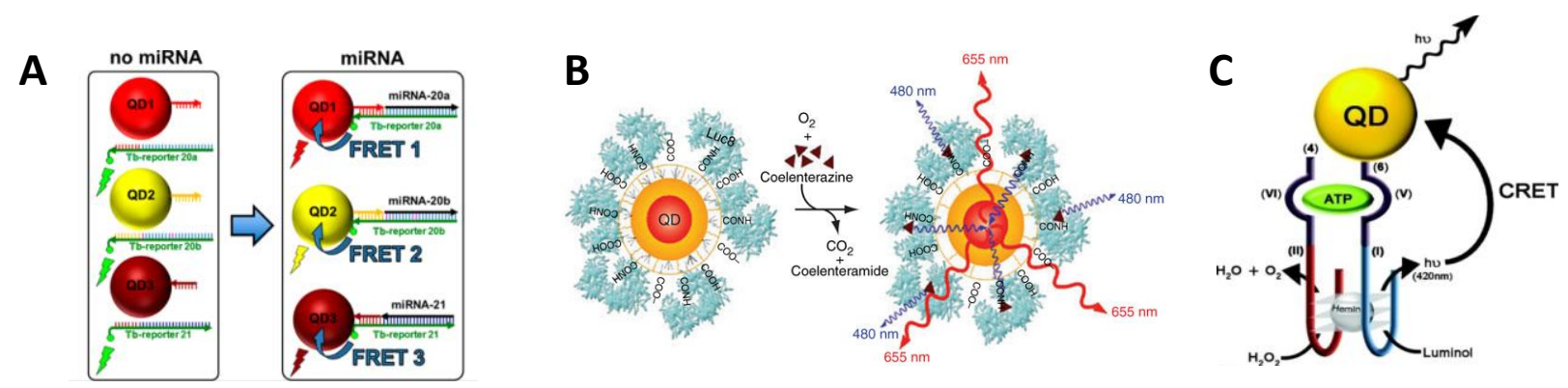

Figure 10. (A) FRET configuration using Tb-complex donor and QD acceptors applied in a multiplexed assay using three different QDs to detect multiple miRNAs. The sandwich hybridization made of three DNA strains: short complementary DNA strain conjugated on the QD, Tb-labeled DNA strands (Tbreporter) and target miRNAs. This results in close proximity and enables FRET interactions. (B) Schematic representation of a QD conjugated to a BRET donor, Luc8. The bioluminescence energy produced by Luc8-catalyzed oxidation of coelenterazine is transferred to the $Q D$, resulting in $Q D$ emission. (C) Schematic illustration of a sensor assembling based on CRET. The sensor detects ATP through the CRET from luminol, oxidized by the assembled hemin/G-quadruplex, to the QDs. Figures reprinted from references, $[161,162,164]$ with permission of the American Chemical Society and NPG. 
A

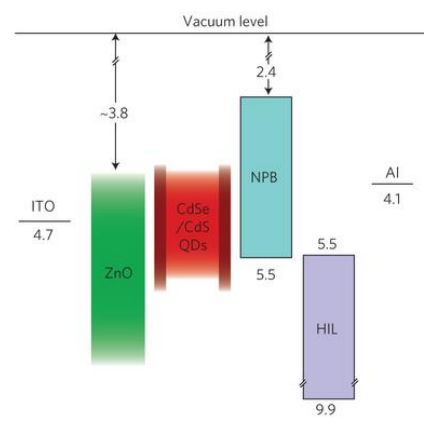

B

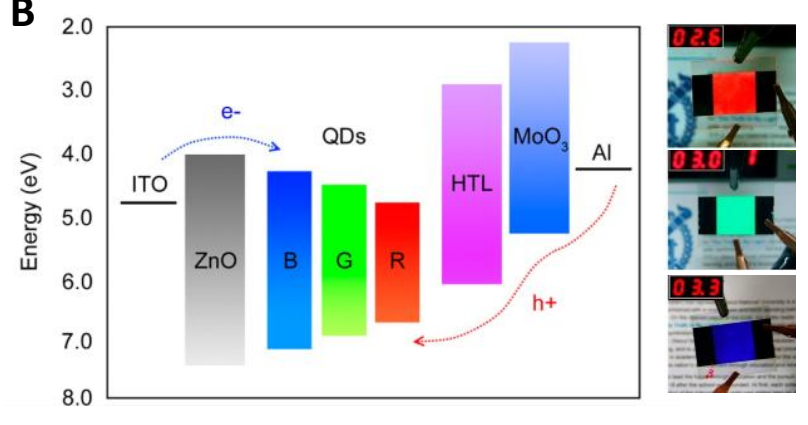

C (i)

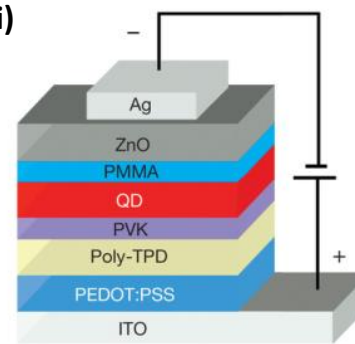

D (i)

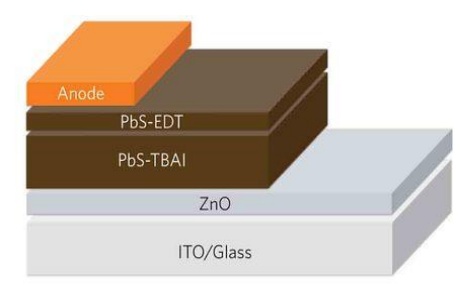

(ii)

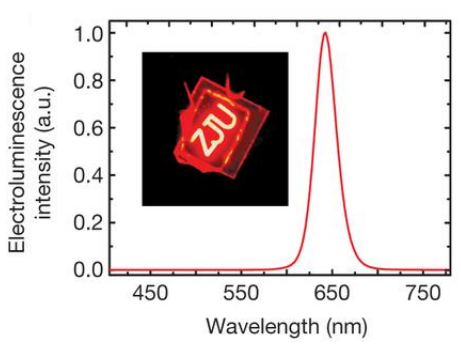

(ii)

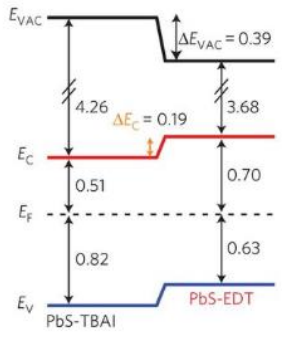

(iii)

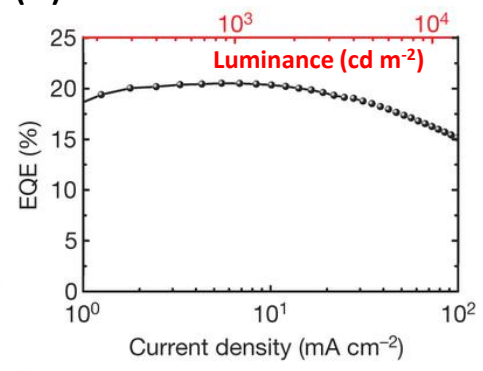

(iii)

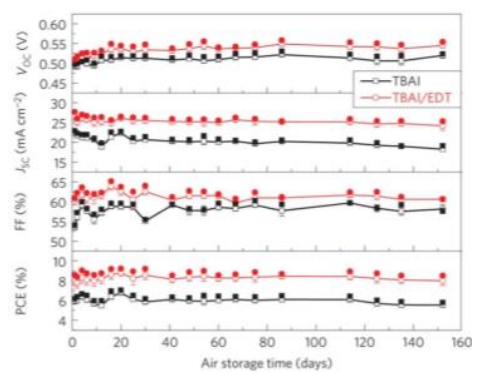

Figure 11. (A) Energy diagram of the high-efficiency QD-LEDs detailing the various layers; layers were fabricated via spin-coating the electron transport layer of $\mathrm{ZnO}$ nanocrystals, the layer of $\mathrm{QD}$ emitters, followed by thermal-evaporation deposition of the hole transport and hole injection layers. (B) A schematic representation of the energy band diagram of full-color QD-LEDs in the unbiased condition. Contrary to a conventional device structure, the electrons are injected from ITO, while holes are injected from Al (i.e., inverted structure). (C) Multilayer QD-LED: (i) device structure containing an insulating PMMA layer inserted between the QD layer and the ZnO layer; (ii) normalized electroluminescence spectrum collected using an applied voltage of $3 \mathrm{~V}$; (iii) external quantum efficiency vs. current density and luminance for the device with best performance. (D) QD solar cell: (i) photovoltaic device architectures with n-type PbS-TBAI and p-type PbS-EDT; (ii) schematic energy level alignment at PbS-TBAI and PbS-EDT interfaces; (iii) evolution of the photovoltaic parameters collected for PbS-TBAl (black) and $\mathrm{PbS}-\mathrm{TBAI} / \mathrm{PbS}$-EDT (red) devices. Figures adapted from references,[165-168] with permission of the American Chemical Society and NPG. 


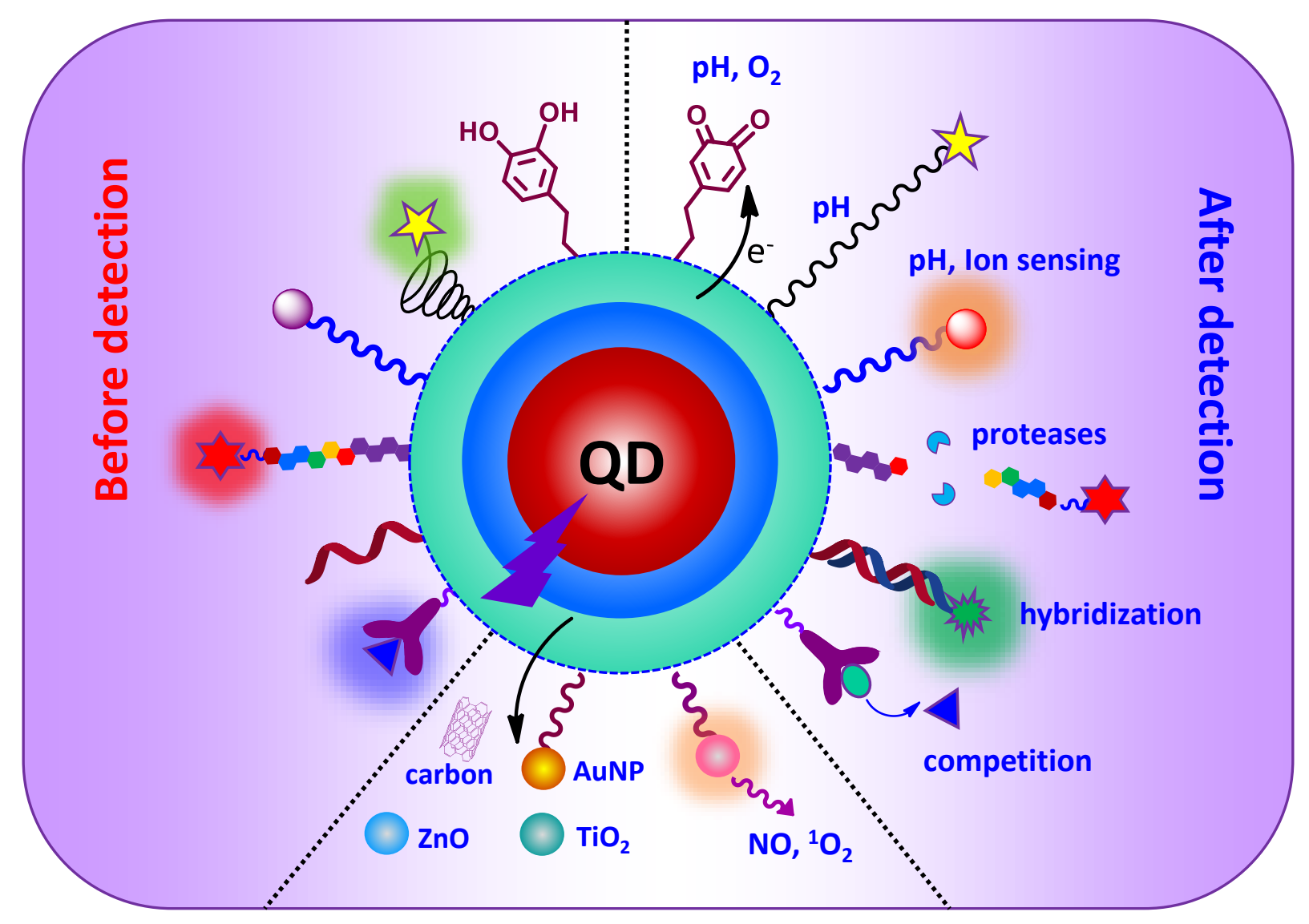




\section{Biographies:}

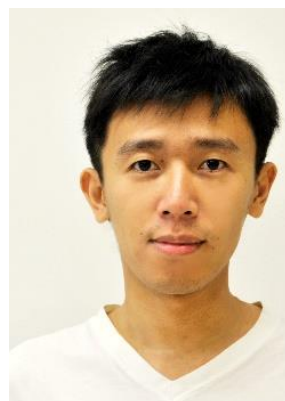

Xin Ji received his bachelor's and master's degrees in chemistry from Jilin University, China. He obtained his PhD in Material Chemistry from Florida State University under the supervision of Professor Hedi Mattoussi in the spring of 2015. His PhD research focuses on the development of a novel sensing platform made of luminescent quantum dots covalently coupled to a controllable number of dopamine groups. He carried out a systematic investigation of the effects of solution $\mathrm{pH}$, oxygen, $\mathrm{QD}$ energy bandgaps and the separation distance on the charge transfer interactions in such assemblies. $\mathrm{He}$ is currently working as a research scientist at the Ocean NanoTech, LLC. His research interests lay at the interface of nanomaterials and biotechnology with an aim towards disease diagnostics and therapeutics.

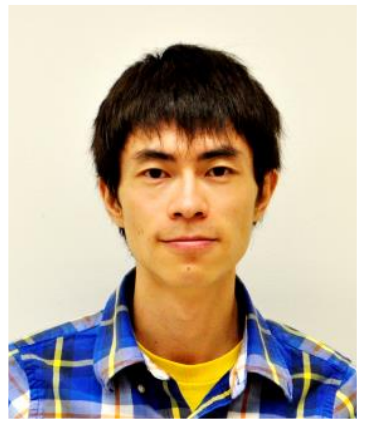

Wentao Wang received a Bachelor degree in Chemistry from Jilin University, China. He then joined the Florida State University in the fall of 2011. He has been a Ph.D. candidate under the supervision of Prof. Hedi Mattoussi, in the Department of Chemistry and Biochemistry since January 2012. His research focuses on the design of multidentate and multifunctional polymer ligands readily adaptable various metal-rich surfaces, including QDs, metal and metal oxide nanocrystals. He is also developing novel approaches for surface modification of inorganic nanocrystals and the development of biological sensing and imaging using these optimally designed nanoprobes.

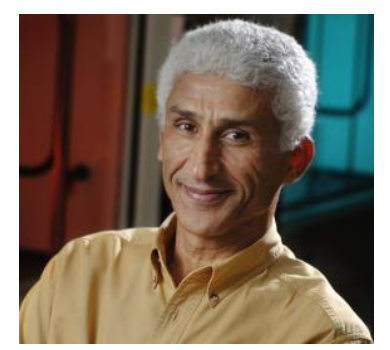

Hedi Mattoussi has been a professor at the Florida State University, Department of Chemistry and Biochemistry, since August 2009. Prior to that, he spent 12 years working as a senior Scientist at the Naval Research Laboratory in Washington, DC. He received a bachelor in Physics from the Faculty of Sciences in Tunis and a Ph.D. in Condensed Matter Physics from the University of Pierre \& Marie Curie (Paris VI) in 1987. In 1994, he received a Habilitation to direct Research, Materials Physics, also from the University of Pierre and Marie Curie. He presently focuses on interfacing inorganic nanoparticles with biological systems using chemical and photochemical means, to develop novels tools for imaging, sensing and diagnostics. Mattoussi is Fellow of the American Chemical Society, class of 2011, the American Physical Society, class of 2014, and the Royal Society of Chemistry (UK), class of 2015. 